\title{
Les cadres surchargés par leurs emails : déploiement de l'activité et expérience vécue
}

Managers swamped by emails: their deployed activity and experiences

\author{
Lisa Créno et Béatrice Cahour
}

\section{(2) OpenEdition}

\section{Journals}

Édition électronique

URL : http://journals.openedition.org/activites/2698

DOI : 10.4000/activites.2698

ISSN : 1765-2723

Éditeur

ARPACT - Association Recherches et Pratiques sur les ACTivités

Référence électronique

Lisa Créno et Béatrice Cahour, « Les cadres surchargés par leurs emails : déploiement de l'activité et expérience vécue », Activités [En ligne], 13-1 | 2016, mis en ligne le 15 avril 2016, consulté le 30 avril 2019. URL : http://journals.openedition.org/activites/2698; DOI : 10.4000/activites.2698

Ce document a été généré automatiquement le 30 avril 2019

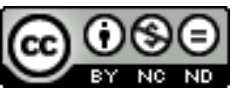

Activités est mis à disposition selon les termes de la licence Creative Commons Attribution - Pas d'Utilisation Commerciale - Pas de Modification 4.0 International. 


\title{
Les cadres surchargés par leurs emails : déploiement de l'activité et expérience vécue
}

Managers swamped by emails: their deployed activity and experiences

\author{
Lisa Créno et Béatrice Cahour
}

\section{NOTE DE L'ÉDITEUR}

Article soumis le 11 février 2015, accepté le 17 janvier 2016

\section{Introduction}

1 Les travailleurs et en particulier les travailleurs de l'information voient leur travail s'intensifier. Selon Théry (2006), l'intensification du travail renvoie à l'augmentation de la pression au travail, résultant de la combinaison de contraintes tayloriennes, de formes évènementielles liées au travail en flux tendu, de contraintes marchandes découlant de la recherche de la satisfaction du client, de tensions entre objectifs et procédures, entre les prescriptions et les réalisations. Elle s'observe lorsque les contraintes de travail, et surtout la pression temporelle, restreignent les espaces de liberté de l'individu. Les Nouvelles Technologies de l'Information et de la Communication (Gueguen, 1997) proposaient d'améliorer la communication entre les équipes, de répondre plus rapidement aux demandes, de se tenir informé sur de nombreux domaines d'activités. En un mot, d'être plus réactif et plus compétitif. Les sociétés industrielles ont alors rapidement misé sur l'implantation massive d'un nouvel outil de communication: la messagerie électronique. Initialement très prisé pour ses avantages d'immédiateté et de bas coût, l'email était annoncé comme facteur de développement social et de progrès (Jauréguibéry, 2012), censé améliorer les qualités de communications, productions, 
collaborations, prises de décision, des individus et des collectifs (Ducheneaut \& Belloti, 2001).

Depuis la diffusion et la généralisation des usages de l'email dans toutes sortes d'organisations, son usage excessif est à l'origine d'importantes problématiques managériales et psycho-sociales (Tahri \& Fallery, 2010). Plusieurs études font état des liens de causalité existants entre l'usage de l'email et différents types de risques psychosociaux tels que le stress au travail (De la Rupelle, Fray, \& Kalika, 2014 ; Mark, Voidam, \& Carello, 2012 ; Renaud, Ramsay, \& Hair, 2006), les violences verbales et harcèlement médiés (Gauducheau, 2012), les phénomènes de surcharge (Bobillier-Chaumon, \& Triposelli, 2012 ; Whittaker \& Sidner, 1996), d'intensification du travail (Klein \& Ratier, 2012) ou encore de fragmentation des tâches (Assadi \& Denis, 2005) chez les usagers professionnels. Les contraintes à la fois organisationnelles, cognitives et émotionnelles imposées par une gestion quotidienne des emails entraînent des expériences utilisateurs marquées de souffrances professionnelles encore peu renseignées.

3 L'article vise à renseigner les caractéristiques de cette activité située de gestion des emails et les expériences vécues associées de cadres surchargés par leurs emails. Grâce à la mise en place d'une méthodologie de recherche originale, articulant les points de vue intrinsèque et extrinsèque situé et non situé de l'activité (Créno \& Cahour, 2015), nous répondrons plus précisément aux questions suivantes : quelles sont les sources à l'origine de ce sentiment de surcharge d'emails? Quels sont les effets délétères pour le bien-être au travail de ces cadres et quelles sont les émotions négatives engendrées par cette activité? Comment les usagers surchargés tentent-ils de faire face à cette surcharge d'emails et notamment quelles activités quotidiennes de lecture/traitement/rédaction d'email mettent-ils en place? Les cadres usent-ils de stratégies particulières pour surmonter cette surcharge et les difficultés associées? À partir de cette analyse d'activité, quelles recommandations peut-on proposer pour les cadres concernés?

4 L'article présentera, dans un premier temps, un état de l'art sur l'intensification du travail et les impacts cognitivo-émotionnels engendrés par l'usage de la messagerie électronique; dans un second temps, les objectifs de l'étude et la méthodologie déployée seront précisés; et enfin, les résultats portant sur l'analyse plus spécifique de 4 cadres surchargés par leurs emails seront exposés puis discutés au regard de la littérature.

\section{2. État de l'art sur l'intensification du travail et les impacts cognitivo-émotionnels engendrés par l'usage de la messagerie électronique}

5 L'usage de l'email est intrinsèquement lié au contexte professionnel des cadres. Nous présentons successivement les caractéristiques et les effets de l'intensification du travail sur les opérateurs, les impacts émotionnels de l'email sur les opérateurs, ainsi que les moyens organisationnels petit à petit imaginés par les organisations pour tenter de diminuer les souffrances professionnelles. 


\subsection{Intensification du travail amplifiée par l'usage de l'email}

\subsubsection{Risques psychosociaux, intensification du travail et $\mathrm{TIC}^{1}$} mutations et tendent vers une généralisation de l'intensification au travail, au travers : la complexification des systèmes et des tâches, l'optimisation voire la suppression des temps morts, l'individualisation des postes de travail, la production en flux tendu pour des rentabilisations maximales, l'augmentation des exigences clientèles, etc. Le taux de salariés victimes de risques psychosociaux ne cesse de croitre, environ $20 \%$ des salariés européens estiment que leur santé est affectée par des problèmes de stress au travail. Les risques psychosociaux recouvrent certaines manifestations comme "le stress, le mal-être, l'inquiétude, la tension pouvant se développer sous des formes aggravées (angoisse, souffrance, épuisement au travail - burn-out, dépression, somatisation...) et pouvant donner lieu à différents types de comportements (agressivité, comportements violents, conduites addictives, harcèlement) affectant la sphère intime du salarié et/ou les relations entre les individus » (De Gasparo \& Van Belleghem, 2013). Si ces risques sont parfois délicats à identifier, du fait de leurs dimensions individuelles et subjectives, l'organisation doit y être attentive et chercher à identifier les sources à l'origine de leur développement.

7 L'usage de plus en plus complexe des TIC et de l'email, par les «contraintes de gestion invisibles» (de Terssac, 1990, p. 137) infligées aux utilisateurs peuvent apparaître comme l'une des sources de pénibilité. La messagerie électronique contribue notamment à accroitre le volume et le temps passé à travailler (Barley, Meyerson, \& Grodal, 2011). Des chiffres alarmants conduisent d'ailleurs les organisations à s'interroger davantage sur les enjeux de l'utilisation de la messagerie électronique. Mano et Mesch (2010) indiquent qu'en Angleterre, chaque opérateur passe en moyenne 5 minutes pour traiter un email et comme il en reçoit environ 39 par jour, il passe plus de 3 heures par jour pour les gérer. De la même façon, il y a déjà 10 ans, Whittaker et Sidner (1996) (2001) rapportaient que $32 \%$ des managers souffraient d'une invasion d'emails impactant négativement leur activité professionnelle. Le problème de gestion des emails s'étend à un nombre croissant d'opérateurs : $56 \%$ des opérateurs en France (toutes professions confondues) consacrent d'ailleurs plus de 2 heures par jour à la gestion de leur messagerie électronique ; $38 \%$ des utilisateurs reçoivent plus de 100 messages par jour, à la pertinence plus ou moins élevée (ORSE, 2011). Aussi, $70 \%$ des managers disent "souffrir de surcharge informationnelle» directement causée par l'abondance et la non-pertinence des emails gérés. La messagerie est donc également à l'origine de surcharges informationnelle et communicationnelle, et plus généralement d'un syndrome de débordement cognitif, décrits ci-dessous.

\subsubsection{Surcharges et débordement cognitif}

Les opérateurs verbalisent beaucoup sur cette surcharge informationnelle induite par la messagerie, car ils constatent que les informations reçues sont de plus en plus quantitativement importantes, créant un sentiment de «brouillard informationnel » (Shenk, 1998). La surcharge communicationnelle, quant à elle, peut être définie comme un excès de sollicitations non pertinentes pour la tâche en cours (Assadi \& Denis, 2005), pouvant entraîner des échanges d'emails jugés inutiles ou "pourriels ». En effet, si l'email est considéré comme peu dérangeant et peu perturbant pour l'émetteur, il l'est en revanche beaucoup plus pour le récepteur qui semble presque contraint de réceptionner ses mails 
au fil de l'eau, augmentant ainsi le nombre d'interruptions de tâches. Pour Kalika, Boukef Charki et Isaac (2007), ces phénomènes s'accentuent lorsqu'il y a dans l'entreprise et pour l'opérateur, une multiplication de médias de communication (messagerie électronique, intranet, messagerie instantanée, réseau social) et de supports d'informations temps réel, il nommera cela la «théorie du millefeuille ", désignant l'empilement des effets néfastes dus à l'empilement de différents canaux de communication utilisés dans l'entreprise.

Lorsque l'opérateur subit un coût excessif et une saturation d'informations et de communications, il peut être atteint du syndrome de débordement cognitif ou «COS " (Cognitive Overflow Syndrom) mis en exergue par Lahlou (2000), qui désigne la forme pathogène du débordement cognitif. Le « $\operatorname{COS}$ » concerne particulièrement les opérateurs surchargés par leur messagerie électronique et provient de l'association d'un ou plusieurs des facteurs suivants: (1) une production croissante d'informations, en volume, les flux de messages, notamment en courrier interne ; (2) un stress des individus, qui se plaignent d'être "débordés ", "noyés ", par une information «inutile ", qu'ils n'arrivent pas à traiter, et qui s'empile. Les plaintes se focalisent sur le manque de temps et le retard dans l'avancement du «vrai » travail, qui serait empêché par des opérations de traitement d'information inutile ; (3) l'impossibilité d'attribuer une cause unique au phénomène ; (4) la perte de sens, les individus n'arrivent plus à produire du sens à partir de l'information, et se réfugient dans des stratégies de court terme. Ces différents phénomènes impactent fortement l'activité cognitive et émotionnelle des usagers concernés.

\subsection{Impacts émotionnels de l'usage de l'email au travail}

\subsubsection{Phénomènes de dispersion, tension et pression temporelle}

10 L'utilisation de la messagerie électronique en fond d'activité professionnelle impose aux opérateurs une attention partagée continue, souvent jugée inconfortable. Datchary et Licoppe (2007) ont montré que cette dispersion au travail induisait une charge cognitive non négligeable, difficilement maîtrisable et maîtrisée par l'opérateur. La "présence obstinée » des emails et les alertes visuelles ou sonores qui y sont associées contribuent à attirer l'attention de l'opérateur et à l'absorber dans une tâche qu'il n'avait pas considérée comme prioritaire. Les auteurs parlent d'ailleurs de possible apparition de " tensions qui pèsent globalement sur l'activité » de l'opérateur. Lee et Pantelli (2011) montrent que le média peut être utilisé de manière excessive et introduire de la pression dans la résolution des problèmes de délai ou pour faire un point sur l'avancement d'un document. Cette pression exercée par l'email organise un rapport au temps considéré comme flux « continu » permanent, contradictoire avec le temps réel dans lequel évolue l'opérateur, l'obligeant à se resynchroniser régulièrement, entraînant également une porosité des frontières travail-hors travail (Bretesché, de Corbière, \& Geoffroy, 2012). L'accessibilité, l'instantanéité et l'obligation implicite de répondre aux emails à toutes heures entraînent même chez certains opérateurs une "overdose communicationnelle» selon l'expression de Jauréguiberry (2010). Plusieurs travaux ont montré les liens existants entre la messagerie électronique et le sentiment d'urgence des opérateurs. Le média accélère en effet les rythmes de travail et exige des temps de réponses toujours plus courts (Taylor, Fieldman, \& Altman, 2008). Certains auteurs parlent même d'un « état d'urgence » (Blanc \& Ettighoffer, 2002). D'un côté, l'email impose une accélération du temps de réponse et de l'autre, il ralentit l'activité de «vrai » travail telle que l'entend l'opérateur. C'est ce phénomène qui contribue à augmenter le sentiment de stress. 


\subsubsection{Stress et autres émotions négatives engendrés par l'usage de l'email}

11 La technologie de communication et d'information altère significativement les conditions de travail, en augmentant l'intensification du travail, qui en conséquence contribue à générer du stress ${ }^{2}$ et de la tension chez les opérateurs (Chesley, Moen, \& Shore, 2003). Renaud et al. (2006) décrivent un phénomène de «stress numérique» ou encore de «stress de l'e-mail». Une étude consistant à priver les opérateurs de messagerie électronique pendant 5 jours permetent à Mark et al. (2012) de tester les effets de la déconnection chez des utilisateurs d'emails. Il en ressort notamment une diminution notable du stress (relevé grâce à des mesures physiologiques - mesures des catécholamines) et ce, dès le premier jour d'arrêt de la gestion des emails professionnels. Mais on se questionne alors sur les effets post-déconnexion induits chez ces cadres, lorsque la gestion des emails doit reprendre, comment se sentent-ils à l'ouverture d'une messagerie dont le flux d'emails entrants lui, ne s'est pas interrompu?

Enfin, dans une récente étude menée auprès de 25 utilisateurs de messagerie électronique, Gauducheau (2012) identifie les principaux affects engendrés plus ponctuellement par la gestion des emails :

- La colère provient surtout de l'évaluation négative que les utilisateurs font des messages reçus. Ce sont des emails qui ne correspondent pas à la forme syntaxique attendue par les utilisateurs. Ces derniers parlent alors de "violations des règles sociales et communicationnelles " ou de «manque de savoir-communiquer» selon une expression de Marcoccia (1998, cité par Gauducheau, 2012). Le manque de soin dans le style rédactionnel ou la qualité de l'orthographe en font partie. La formulation d'un ordre «brutal » peut aussi générer colère ou irritation.

- L'irritation vient principalement du caractère «intrusif» de l'email. C'est aussi du mécontentement qui ressurgit chez les utilisateurs de messagerie qui parlent avant tout de la peur d'être débordé par le nombre d'emails, par la gêne occasionnée par ces interruptions sans fin et peu maîtrisées, par le fait que les emails sont de plus en plus intrusifs dans la vie privée de ces utilisateurs. Les auteurs montrent que la réception des emails est souvent vécue comme contraignante et pesante. Certains parlent de désagrément, d'autres évoquent même le débordement.

13 La peur, dont les principales causes seraient le manque de compétence dans la maîtrise de l'outil informatique ou encore le manque de repères et de règles lors de la production d'emails (syntaxe, orthographe, possible incompréhension). Malgré ces premières études à visée qualitative, les connaissances de l'activité réelle des usagers de l'email restent limitées. La prise de conscience du problème se fait alors progressivement, avec la mise en place et les essais de plusieurs types de recommandations.

\subsection{Moyens organisationnels mis en place}

14 La médecine du travail, les représentants du personnel et des syndicats, la direction des ressources humaines ou de la communication peuvent être à l'origine de différents moyens de cadrage de l'activité de gestion des emails. Ci-dessous, quelques-uns de ces moyens, nous commentons leurs limites. 


\subsubsection{Cadrer les pratiques de gestion des emails} présentées sous forme de "règlement officiel de la bonne communication", listant différents principes de précautions à l'égard de ses utilisateurs. Les guides tentent de faire oublier le manque de règles de communication par email en espérant apprendre aux utilisateurs comment limiter les pertes de temps, la dépendance, la surcharge informationnelle, les conflits et le stress lors de la gestion des emails. Ces chartes, généralement établies par l'organisation seule, de manière unilatérale et générale, recensent les bons usages sans tenir compte des réalités socioprofessionnelles et/ou des spécificités des métiers. Ces «bonnes pratiques » deviennent de nouvelles règles et de nouvelles prescriptions qui peuvent avoir pour objectif de dédouaner l'organisation en cas de problèmes (au niveau des risques psycho-sociaux et du débordement hors-travail, notamment). À notre connaissance, elles n'ont pas réussi à gommer les effets néfastes de la messagerie.

Quelques entreprises ont organisé des séances de retours d'expériences et des formations destinées aux managers les plus surchargés par leurs emails. L'objectif avancé, rapporté par quelques cadres de notre panel, est notamment de les accompagner dans leur gestion quotidienne des emails, en encourageant le partage d'expérience ou en illustrant les «bonnes pratiques » listées sur les chartes présentées précédemment. Nous savons peu de choses sur les opérateurs qui ont accepté de participer à ces formations, le contenu qui y était présenté, ou encore les bénéfices obtenus à court et moyen termes.

Pour donner quelques exemples, en 2010, France Télécom crée un accord sur l'équilibre vie privée-vie professionnelle (5 mars 2010) : «De façon à prévenir de l'usage de la messagerie professionnelle, le soir, le week-end et pendant les congés, il est rappelé qu'il n'y a pas d'obligation à répondre pendant ces périodes et qu'il est recommandé d'utiliser les fonctions d'envoi différées »; Air France et Sneda $(2009,2006)$ proposent également un accord sur la mise en place du télétravail dans l'entreprise, fixant les conditions de joignabilité de l'utilisateur (pas de contact possible en dehors des plages définies par l'utilisateur et son responsable) et les délais de réponse en cas de sollicitation par email (s'il y a sollicitation pendant la journée de travail, l'utilisateur a l'obligation de répondre dans la demi-journée). La réussite de ces règles de cadrage réside dans la participation strictement collective de l'ensemble de l'entreprise aux nouvelles règles de communication.

\subsubsection{Encourager la déconnexion}

Depuis peu, des conduites de déconnexion apparaissent à l'initiative des organisations et/ ou des usagers eux-mêmes, encourageant à " reprendre souffle et distance " (Jauréguiberry, 2012) en se coupant, de façon ponctuelle et souvent partielle, du flux informationnel et communicationnel, transitant par email. Depuis 2012, ATOS, Siemens et Total tentent d'encourager la déconnexion partielle de leurs employés, en les privant par exemple de leur smartphone de 18 à 7 heures, bloquant ainsi toute réception ou tout envoi d'email. Nous manquons aujourd'hui de recul pour connaître les effets bénéfiques de telles mesures. D'ailleurs, Jauréguiberry (2012) souligne que «si ses pratiques existent bien, elles sont bien moins importantes que leur représentation: on parle plus de déconnexion que l'on se déconnecte». 
19 Malgré cet aperçu des travaux menés sur les TIC et les impacts négatifs des usages de l'email sur le bien-être au travail des utilisateurs connectés, de nombreuses questions restent en suspens, concernant notamment: les origines précises de cette gestion débordante d'emails; les caractéristiques de la gestion quotidienne d'email dans ses phases de lecture, classement et rédaction; les expériences vécues des usagers en prise avec leur messagerie électronique.

\section{Questions de recherche et méthodologie}

Notre problématique de recherche vise à renseigner le déploiement de l'activité de gestion des emails chez un panel de cadres surchargés par leurs emails et à accéder aux expériences et émotions négatives vécues par ces cadres. L'objectif implicite est de parvenir à comprendre les dangers réels de cette activité quotidienne. Notre étude est orientée vers une analyse principalement "située ${ }^{3}$ " et écologique ${ }^{4}$ des usages et des expériences vécues de cadres surchargés, elle vise également quelques généralisations de phénomènes et procédures typiques à partir de recueils plus quantitatifs. Les questions de recherche plus précises sont présentées lors du développement de la méthodologie.

\subsection{Recueils de données : triangulation des méthodes et des données}

21 Pour parvenir à se saisir de la complexité de cet objet d'étude, à la fois difficile d'accès par sa dimension confidentielle, son déploiement spatial et temporel étendu, et à cause du sentiment de « honte » qui envahit parfois certains utilisateurs débordés par leurs emails, une triangulation des méthodes a été mise en place $^{5}$. Selon Fortin (1996, p. 318), la triangulation correspond «à l'emploi d'une combinaison de méthode et de perspectives permettant de tirer des conclusions valables à propos d'un même phénomène ». Dans notre cas, l'approche adoptée est centrée sur l'expérience vécue des utilisateurs et leur activité réelle et fournit des données qualitatives et quantitatives, avec des descriptions de l'activité du point de vue des sujets (entretiens d'explicitation et d'auto-confrontation, carnets de bord) et du point de vue de l'observateur (journées d'observation). L'« expérience vécue " renvoie à des approches d'ordre phénoménologique telles que Vermersch et Theureau ont pu les développer (cf. Cahour, Salembier, \& Zouinar, 2016, Zouinar \& Cahour, 2013), elles donnent la priorité au point de vue subjectif (ou « intrinsèque », selon Theureau, 2002) recueilli au travers de descriptions fines des vécus tels qu'ils se sont déroulés en situation (et non au travers d'une position analytique et explicative) et qui considèrent les aspects sensoriels et émotionnels de l'action « incarnée ».

\subsubsection{Sélection de deux panels de cadres aux niveaux de surcharge hétérogènes}

Deux panels de cadres ont été sélectionnés pour répondre aux exigences méthodologiques. La taille des panels ainsi que les niveaux de surcharge des cadres varient. 


\subsubsection{Panel 1 : pour une étude située de l'activité et des expériences subjectives}

Dans cet article, nous nous centrerons sur l'analyse de l'activité et des expériences vécues de 4 cadres s'estimant particulièrement "surchargés » par leurs emails. Ils convoquent des spécificités d'usages et des vécus subjectifs différents qui nous semblent intéressants de présenter et de mettre en regard. Ces 4 cadres sont issus d'un premier panel de 9 cadres s'estimant «surchargés " par leurs emails. Les caractéristiques de ce premier panel sont détaillées dans le tableau ci-dessous (Figure 1). Nous distinguons 4 femmes, 5 hommes, âgés de 25 à 57 ans (moy: 43 ans), ils ont un statut de cadre et exercent dans des domaines variés (technique-informatique, énergie, recherche publique, commerce). Leurs contacts sont majoritairement étendus à l'international. Ils travaillent dans des environnements hyper-connectés, ont plusieurs supports de gestion d'email. Bien que la constitution de ce panel et la proposition de notre suivi méthodologique n'aient pas été aisées, pour des questions de temps disponible, de honte ou de confidentialité professionnelle, la réflexivité potentiellement apportée aux cadres surchargés était un sérieux argument pour leur participation volontaire. Nous présentons ci-dessous leurs caractéristiques individuelles et résumons en premier lieu les contextes socioprofessionnels des 4 cadres que nous présentons plus précisément dans cet article.

Figure 1 : Panel $1: 9$ cadres surchargés observés et interviewés pour l'étude (les prénoms ont été modifiés).

Figure 1: Panel 1: 9 overloaded managers observed and interviewed for the study (their names have been changed)

\begin{tabular}{|c|c|c|c|c|c|c|c|}
\hline Prénom & $\begin{array}{l}\text { Âge } \\
\text { (ans) }\end{array}$ & Fonction & $\begin{array}{l}\text { Type de } \\
\text { contacts }\end{array}$ & Env. de travail & Equipement TIC & $\begin{array}{l}\text { Messagerie et } \\
\text { types d'emails }\end{array}$ & $\begin{array}{c}\text { Alertes sonores } \\
\text { ou visuelles } \\
\text { quand réception } \\
\text { d'un email }\end{array}$ \\
\hline Albert (Al) & $45-55$ & $\begin{array}{c}\text { Dir. } \\
\text { Technique }\end{array}$ & Inter. & $\begin{array}{l}\text { Bureau seul, } \\
\text { vitres } \\
\text { transparentes }\end{array}$ & $\begin{array}{l}\text { PC portable, fixe, tél. } \\
\text { fixe et Smartphone }\end{array}$ & Outlook, pro & Oui \\
\hline \multicolumn{8}{|c|}{ 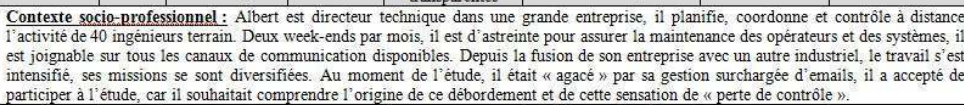 } \\
\hline $\begin{array}{l}\text { Géraldine } \\
\text { (G) }\end{array}$ & $>55$ & Dir. Projet & Nat. & Bureau à 2 & $\begin{array}{l}2 \text { PC portable, fixe, } \\
\text { tel. fixe et portable, } \\
\text { MI, reseaux sociaux, } \\
\text { plateforme }\end{array}$ & $\begin{array}{l}\text { Lotus Notes, } \\
\text { perso et pro }\end{array}$ & Oui \\
\hline \multicolumn{8}{|c|}{ 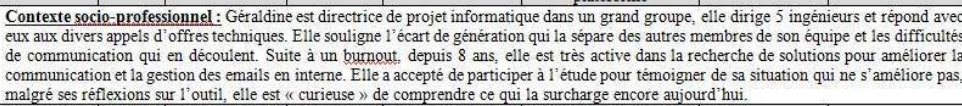 } \\
\hline Anna (A) & $35-45$ & Dir. Securité & Inter. & $\begin{array}{l}\text { Bureau seul, } \\
\text { vitres } \\
\text { transparentes }\end{array}$ & $\begin{array}{c}\text { PC portable, fixe, tél. } \\
\text { portable et fixe }\end{array}$ & Outlook, pro & Oui \\
\hline \multicolumn{8}{|c|}{ 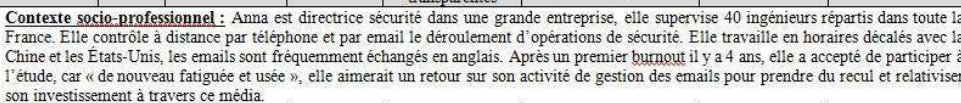 } \\
\hline $\begin{array}{c}\text { Fabien } \\
(\mathrm{Fa})\end{array}$ & $25-35$ & Dir. Financier & Inter. & $\begin{array}{l}\text { Bureau seul } \\
\text { fermé }\end{array}$ & $\begin{array}{c}\text { PC portable, fixe, } \\
\text { Smartphone, tel. fixe }\end{array}$ & $\begin{array}{c}\text { Lotus Notes, } \\
\text { pro }\end{array}$ & Oui \\
\hline \multicolumn{8}{|c|}{ 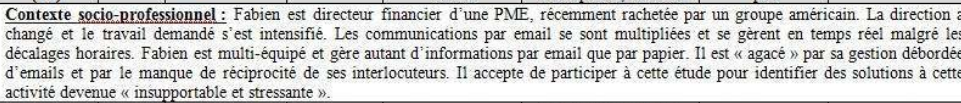 } \\
\hline Iris (I) & $>55$ & Dir. Projet & Nat. & Bureau à 2 & $\begin{array}{l}\text { PC portable, tél. fixe } \\
\text { et portable, MI }\end{array}$ & Outlook, pro & Oui \\
\hline Francis $(\mathrm{Fr})$ & $35-45$ & Dir. Projet & Inter. & $\begin{array}{l}\text { Bureau seul, } \\
\text { vitres } \\
\text { transparentes }\end{array}$ & $\begin{array}{l}\text { PC portable, fixe, tél. } \\
\text { fixe et } 2 \text { Smartphones }\end{array}$ & Outlook, pro & Oui \\
\hline $\begin{array}{l}\text { Mathilde } \\
\text { (M) }\end{array}$ & $35-45$ & $\begin{array}{l}\text { Assistante } \\
\text { direction }\end{array}$ & Inter. & $\begin{array}{l}\text { Bureau à 2, dans } \\
\text { passage }\end{array}$ & PC portable, tél. fixe & Outlook, pro & Oui \\
\hline Charles (C) & $45-55$ & $\begin{array}{l}\text { Dir. Business } \\
\text { Group }\end{array}$ & Inter. & $\begin{array}{l}\text { Bureauge seul, } \\
\text { vitres } \\
\text { transparentes }\end{array}$ & $\begin{array}{l}\text { PC portable, fixe, té. } \\
\text { fixe et Smartphone. }\end{array}$ & Outlook, pro & Oui \\
\hline Jérèmy $(J)$ & $25-35$ & $\begin{array}{l}\text { Enseignant- } \\
\text { Chercheur }\end{array}$ & Nat. & Bureau à 4 & $\begin{array}{l}\text { PC portable, } \\
\text { Smartphone, } \\
\text { télephone, }\end{array}$ & $\begin{array}{l}\text { Outlook, perso } \\
\text { et pro }\end{array}$ & Oui \\
\hline
\end{tabular}

En gris, les 4 cadres que nous détaillerons plus spécifiquement dans cet article. In grey: the 4 managers covered in greatest detail in this article 


\subsubsection{Panel 2 : pour confirmer/infirmer/généraliser les premiers résultats} questionnaire $^{6}$ en ligne que nous avions soumis (construit après analyse des résultats qualitatifs). Les caractéristiques de ce panel sont les suivantes : $40 \%$ de femmes, $60 \%$ d'hommes, l'âge moyen des cadres répondant est de 40 ans, les domaines d'activités ont été classés en 5 catégories: technique-informatique, énergie, commercial, recherche privée et publique, services. Volontairement, nous souhaitions des cadres aux niveaux de surcharge hétérogènes. Parmi les 200 cadres répondants : $10.9 \%$ ne s'estiment «pas du tout surchargés par leurs emails »; $46.3 \%$ ne s'estiment "pas vraiment surchargés par leurs emails »; $35.3 \%$ s'estiment « assez surchargés par leurs emails »; $7.5 \%$ s'estiment «totalement surchargés par leurs emails». Pour cet article, lors des comparaisons de résultats qualitatifs (issus du Panel 1) et quantitatifs (issus du Panel 2), nous retiendrons surtout les données obtenues pour ces 86 cadres $(35.3 \%+7.5 \%)$ s'estimant surchargés.

\subsubsection{Articulation de plusieurs points de vue sur l'activité}

La figure ci-dessous (Figure 2) synthétise la démarche méthodologique de triangulation mise en place pour l'étude des cadres surchargés par leur gestion des emails. Elle présente également les questions de recherche poursuivies.

Figure 2 : Démarche méthodologique globale.

Figure 2: Global methodology

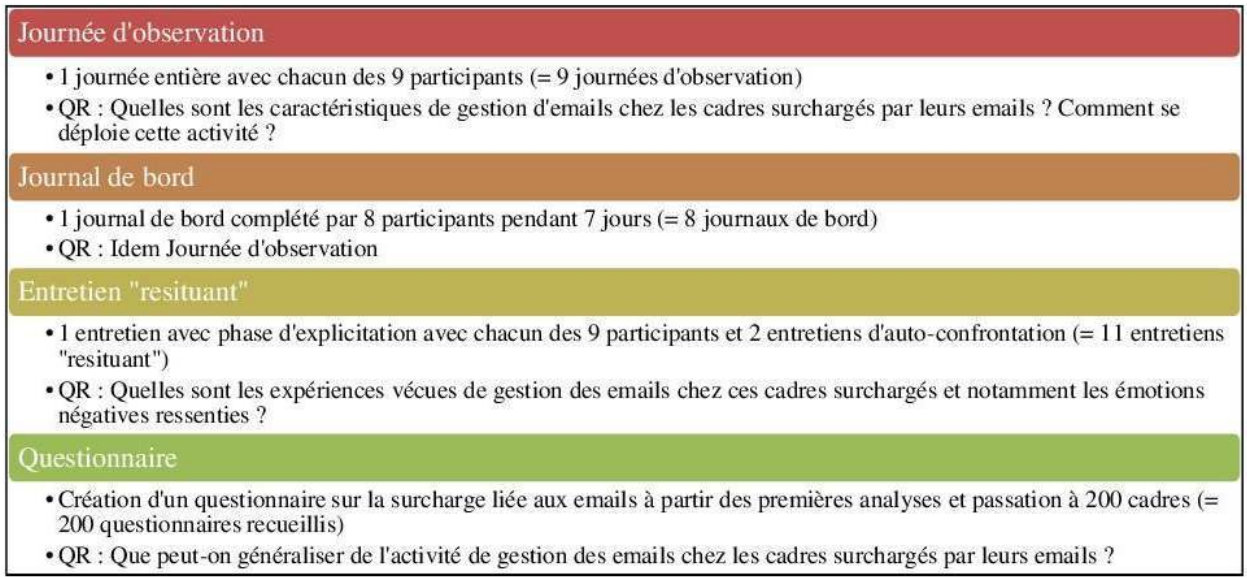

\subsubsection{Journée d'observation}

Suite à une première prise de contact avec les 9 cadres participants, nous avons réalisé 9 journées d'observation (équipés d'une grille d'observables ${ }^{7}$ ), nous permettant de suivre l'activité déployée tout au long de la journée de travail, depuis l'ouverture de la messagerie électronique jusqu'à sa fermeture. Dans la mesure du possible, nous les avons suivis en situation de gestion d'emails "en mobilité ", lors de leurs réunions, pauses, conférences, etc. Nous nous placions à 2 mètres maximum de leur écran pour distinguer et reporter les différentes actions réalisées sur la messagerie. Cette proximité immédiate avec la messagerie du participant était certes inhabituelle, mais nous prenions soin de la négocier avec chacun. Nous n'avons pas encouragé les verbalisations mais les avons 
acceptées lorsqu'elles émanaient d'un besoin du participant. Au total, nous avons recueilli plus de 90 heures d'observation (env. 10 heures / participant), ce qui nous a notamment permis d'explorer les composantes suivantes de l'activité :

- déploiement temporel de l'activité : horaire de première/dernière lecture d'emails, moments d'ouverture/fermeture de la messagerie, temps passé à lire/produire des emails, moments de lectures/production des emails, fréquence de relevé/suppression des emails, etc.

- interruptions de l'activité en cours, causées par les emails entrants ou autres (SMS, collègues, etc.)

- comportements expressifs patents exprimant notamment des situations d'inconfort, plus ou moins manifestes selon les cadres: haussements d'épaules, «hot-sit» (assis-debout sur fauteuil caractéristique de nervosité, Datchary \& Licoppe, 2007), têtes tenues entre les mains, mains levées au ciel, etc.

L'activité de gestion des emails a également été enregistrée grâce à l'installation d'un dispositif vidéo particulier, combinant l'enregistrement des tâches réalisées sur écran (via le logiciel Camtasia) et l'enregistrement du cadre dans son environnement, en point de vue extrinsèque situé (via une caméra posée sur trépied). Deux cadres seulement (le caractère confidentiel du contenu filmé était particulièrement bloquant pour les autres) ont accepté de déclencher ces enregistrements, nous permettant ensuite de procéder à des entretiens d'auto-confrontation.

\subsubsection{Journaux de bord}

Dans la continuité de la journée d'observation, nous souhaitions saisir un aperçu plus longitudinal de l'activité de gestion des emails de ces cadres surchargés et son caractère chronophage sur une période continue de 7 jours, week-ends et soirées compris. Nous avons donc remis à chaque cadre participant un exemplaire vierge du journal de bord ${ }^{8}$, nous permettant de recueillir les informations suivantes:

- nombre moyen d'emails gérés sur 7 jours

- variabilité des lieux et supports de gestion des emails : bureau, domicile, déplacement (voiture, train, avion, en réunion); depuis l'ordinateur portable, le smartphone ;

- activité de gestion des emails durant les soirées et weekends ;

- nombre d'emails " problématiques » gérés : nous laissions le soin à chaque cadre de définir leur type d'emails problématiques, afin de découvrir ce que cela pouvait évoquer pour eux, subjectivement ;

- moments de surcharge en emails ressentis comme particulièrement intenses par les cadres.

\subsubsection{Entretiens resituants}

29 Certains considèrent que seuls les observables permettent d'accéder à l'action située (Suchman, 1987) telle qu'elle se déploie en situation réelle. Mais on considère ici que certains types d'entretiens permettent de « re-situer » (Cahour \& Salembier, 2012) le sujet dans le contexte de l'activité, de faire en sorte qu'il en retrouve un souvenir vivace, et par-là même de limiter les reconstructions et rationalisations dans sa description du vécu. Nous avons donc mené 9 entretiens approfondis en nous inspirant ${ }^{9}$ de la technique de l'entretien d'explicitation de Vermersch (1994). La durée des entretiens variait entre $1 \mathrm{~h} 30$ et $2 \mathrm{~h} 45$ selon la disponibilité des cadres, leur aisance à décrire leur expérience vécue, la réussite de la mise en évocation, et le nombre d'éléments à expliciter. Les entretiens ont été enregistrés puis intégralement retranscrits; pour fluidifier la lecture 
de certaines verbalisations citées dans le texte, les répétitions et marques d'hésitations ("euh::») ont été supprimées. Ces entretiens nous ont alors permis d'approfondir l'expérience vécue des cadres surchargés et notamment certains moments spécifiques repérés en journée d'observation ou ce qui constituaient pour eux des emails "problématiques» (nous leur laissions le choix de définir ce qui pour eux, semblait être un email problématique, au sens de "prise de tête, délicat, compliqué » à gérer) et les émotions associées à leur gestion. Dans la continuité des premiers entretiens avec phases d'explicitation, nous avons ensuite mené 2 entretiens d'auto-confrontation avec des relances inspirées de l'entretien d'explicitation (Theureau, 2002 ; Cahour, 2012) auprès des deux seuls cadres ayant accepté que leurs activités et leurs échanges d'emails soient filmés.

\subsubsection{Questionnaires}

30 Nous avons ensuite, à partir des résultats qualitatifs, créé un questionnaire ${ }^{10}$, composé de 14 questions (13 à choix multiples et 1 ouverte), portant notamment sur l'organisation des emails professionnels, les emails problématiques, les émotions, les modes de coping ${ }^{11}$ mis en place en situation délicate et enfin la principale amélioration souhaitée sur messagerie. Rempli par 200 cadres, dont 86 cadres s'estimaient surchargés, l'objectif de ce questionnaire était de confirmer ou d'infirmer à plus grande échelle certains phénomènes et procédures précédemment identifiés.

\subsection{Analyse des données}

31 L'analyse de contenu a été systématiquement employée, sauf dans le cas du questionnaire où un traitement statistique a été appliqué. Les informations issues des journées d'observation et des journaux de bord ont été reportées informatiquement dans des tableaux Excel nous permettant de comptabiliser les différents phénomènes caractéristiques de la gestion des emails et de souligner les phénomènes à la marge ou les particularités de certains cadres surchargés. Les entretiens resituants (d'explicitation et d'auto-confrontation) ont été intégralement retranscrits. Puis, après une lecture flottante des expériences vécues relatées, nous avons catégorisé les évènements marquants, les modes de traitements récurrents et ceux plus à la marge pour rendre compte des usages réels des différents profils de cadres surchargés (Bardin, 1977).

Cet article met en avant 4 cadres du panel 1 de 9 cadres présenté précédemment. Plusieurs raisons motivent notre choix de présenter leurs cas respectifs : ce sont les cadres les plus surchargés du panel 1, ils représentent toutes les tranches d'âges répertoriées dans le panel 1 , ils occupent des fonctions différentes, ils présentent des motivations différentes dans leur participation à l'étude, enfin ils emploient divers mécanismes de réaction et de gestion de leur surcharge d'emails. Les divergences et ressemblances entre chacun seront soulignées et comparées aux résultats issus de l'étude du panel 2 de cadres surchargés.

\section{Résultats}

Les résultats sont exposés suivant 4 parties. Ils résument les caractéristiques principales des contextes socio-professionnels dans lesquels sont immergés les cadres étudiés, 
présentent les raisons et les effets de la gestion continue des emails, les procédures de lecture/classement/rédaction d'email et les exigences implicites, le fardeau des emails problématiques et enfin les pistes de recommandations proposées.

\subsection{Contextes socio-professionnels des 4 cadres étudiés} cadres observés. Les 4 cadres retenus pour cette étude (présentés dans le tableau, partie 3.1.1.1.) effectuent tous un travail de management de personnel. Des changements organisationnels importants sont à l'origine d'une intensification de leur travail et de leurs communications. Ils souffrent d'être surchargés par la gestion de leurs emails, ils se disent tantôt agacés, interrogatifs ou fatigués. Ils cherchent à comprendre comment cette activité s'est imposée aussi subrepticement dans leur travail de communication au point de leur faire perdre le contrôle des tâches en cours. Parmi ces cadres représentant toutes les tranches d'âge observées, différents facteurs peuvent amplifier le sentiment d'une gestion surchargée d'emails: la multiplication des contacts à l'international, l'effet millefeuille (Kalika et al., 2007) provoqué par l'empilement des outils de communication et/ou la généralisation du Smartphone.

\subsection{Gestion continue des emails : effet émotionnel d'un travail sans limite spatio-temporelle}

Le premier phénomène observé chez ces 4 cadres surchargés est la gestion continue de l'email. Sans unité de temps ni de lieu, cette activité est toujours prioritaire par rapport aux autres activités en cours. Les différentes méthodes qualitatives décrites précédemment nous permettent d'identifier les sources à l'origine de cette hyperconnexion, les conséquences négatives engendrées sur le bien-être au travail de ces cadres et les différentes stratégies mises en place pour tenter de faire face à la surcharge.

\subsection{1. a l'origine de la gestion continue des emails : anticiper l'incertitude du futur et se rassurer}

À la différence de l'appel téléphonique qui impose une réponse et une réaction en temps réel des interlocuteurs, l'email était initialement un outil de communication asynchrone, pour lequel il était possible de différer la lecture, le traitement et la réponse aux messages reçus. Aujourd'hui, le nombre d'emails quotidiens et la variété des contenus sont tels, que les 4 cadres peinent à se défaire du rythme imposé par les interactants. Sur une journée de travail, les 4 cadres observés passent plus de $70 \%$ de leur temps sur leur messagerie et gèrent une moyenne de 110 emails ( $\min : 56 ; \max : 175)$, souvent au détriment de ce qu'ils désignent comme leur "vrai travail». Dans leur entreprise respective, aucune norme sociale n'encadre les horaires ou les jours dédiés à l'envoi de ces emails. De plus, les cadres décrivent l'instauration de «jeux de pouvoirs» entre opérateurs qui concourent à l'envoi d'emails en dehors du temps de travail, le weekend ou la nuit, pour manifester aux autres l'importance de leur implication professionnelle. Anna raconte :

«Y'a pas si longtemps, concours auquel je ne participais pas mais je trouvais ça très déplaisant, c'étaient les gens qui surveillaient l'heure à laquelle ils avaient envoyé leurs derniers messages, et c'était à celui qui en enverrait le plus le dimanche, ou le plus la nuit ». 
Si l'appel téléphonique d'un collègue un vendredi soir à 23 heures semble socialement inapproprié, l'email serait pour certain la démonstration d'un engagement salutaire. Pour faire face à cette "techno-invasion" (Popma, 2013) ou plutôt "email-invasion", les cadres observés restent hyper-connectés. Vérifier ses emails en continu constitue un mode de coping centré problème ${ }^{12}$ qui les rassure. Ils tentent de contenir, voire ralentir, la surcharge de travail qui transite par email, de rester informés des incidents potentiels et de contrer l'effet Fear Of Missing Out (FOMO) ${ }^{13}$. Anna témoigne :

«c'est toujours très désagréable d'apprendre par les autres une info cruciale.

J'apprends trop de trucs ailleurs que par les processus normaux d'escalade, alors si en plus je regarde pas mes mails là, je m'en sors plus du tout ».

Mais cette gestion continue ne les satisfait pas, car même poussée à son paroxysme, elle ne permet pas d'atténuer la surcharge vécue par les cadres. Pire, elle devient anxiogène. Fabien décrit cette ambivalence :

«j'ai un mail, alors bam : je regarde, c'est direct, en réunion aussi oui, bah ça va me rassurer dans le sens où je vais effectivement pas avoir l'impression de passer à côté de quelque chose de primordial, bon en même temps, ça peut me foutre un stress aussi : putain merde c'est vrai faut que je fasse ça aussi ! ».

\subsubsection{Conséquences néfastes pour le bien-être de l'individu au travail}

Pour les 4 cadres étudiés, la surcharge d'email s'observe à travers la manifestation de 5 phénomènes : l'allongement du temps de travail et la porosité de la frontière travail-hors travail, la prise de conscience de l'addiction à l'email amplifiée par le Smartphone, le travail de gestion des urgences et le travail réflexif empêché, la mise en place de stratégies pour tenter de faire face au problème de gestion débordante.

\subsubsection{Allongement du temps de travail et porosité de la frontière travail-hors travail}

Pour tenter de gérer leurs volumétries importantes d'emails, les 4 cadres se contraignent à augmenter les amplitudes horaires de présence au travail, dépassant majoritairement les 11 heures quotidiennes travaillées. Ils viennent plus tôt et repartent plus tard du travail, avec pour première et dernière activité, le contrôle de la messagerie électronique. Géraldine raconte :

« je passe mon temps à ça, un mail par ci, un mail par-là, pour ne rien oublier (...) de laisser s'empiler sans dépiler régulièrement, ça me stresse, ça me reste en fond de pensée comme ça, et je peux rien faire d'autres, donc j'mets des coups d'œil permanents si j'travaille sur un doc à côté je fais toujours un petit contrôle ».

Les cadres qui communiquent avec l'international subissent les contraintes inhérentes au décalage horaire et aux emails reçus la nuit ou tôt le matin. Fabien décrit cette situation pénible où, pour avancer dans la gestion de ses emails, il répond à toutes heures, au risque de ne jamais se déconnecter et d'être déçu du manque de réciprocité :

« c'est très lourd mais j'ai pas le choix ! Comment faire autrement ? Je suis le seul à posséder tous les chiffres de la boite! Alors le tort que j'ai c'est que je réponds tout le temps mais je peux pas tout gérer le lendemain matin non plus ».

Anna travaille à domicile pour rédiger certains emails. Son cas illustre la porosité de la frontière travail-hors travail et les effets délétères sur sa motivation:

«si je sais qu'il y a des choses qui méritent du temps, je prépare mes emails à la maison (...) ah c'est pénible hein c'est sûr! J'ai autre chose à faire... sous prétexte qu'on peut joindre les gens n'importe quand, n'importe comment, si vous voulez on charge la mule jusqu'au bout alors bon-là, la mule elle a plus envie quoi ». 


\subsubsection{Prise de conscience de l'addiction}

La participation des 4 cadres à cette étude témoigne de leur prise de conscience d'un problème de surcharge liée aux emails. Mais les journaux de bord ont permis une observation plus directe et quantitative de leur gestion continue et débordante des emails. Voyant les nombreuses interruptions, les horaires et lieux de consultation reportés, les 4 cadres ont verbalisé leur addiction pour cet outil de communication. Albert s'étonne :

«c'est complètement fou ça, je me rends compte que je suis devenu carrément addict à mes emails ».

Cette addiction s'appréhende encore mieux lorsque les cadres rapportent des périodes de privation d'email particulièrement douloureuses ${ }^{14}$. Il raconte son comportement pendant un séminaire d'une journée où le conférencier avait exigé l'extinction des smartphones :

«à la pause, je suis sorti de la salle, tu vois, et j'ai même pas été aux toilettes avant, ni cigarette rien, c'était vite!! vite!! je rallume mon portable, car: mes emails! Alors qu'au fond, je n'attendais rien de spécial, y'avait pas de crise, pas d'inquiétude à avoir, mais dans le doute ".

Pour chaque nouvel email entrant, Anna décrit sa perte de contrôle :

« c'est ma main, elle y va toute seule, ça va extrêmement vite et c'est plus fort que moi ».

Fabien raconte les effets pervers de cette addiction:

« en étant honnête, à certains moments je me dis que (...) d'être tout le temps raccroché à ça, suivre le fil sans relâche comme une drogue (...) ça commence à me faire chier, je vais tout balancer, tout plaquer ! Bon je me dis aussi que c'est un défi aussi et en général bah je relève le défi, mais complètement au détriment de ma vie perso, ça me rend triste parfois ».

Le Smartphone, identifié par Kalika et al. (2007) comme "nouvelle laisse électronique", amplifie encore cette addiction, puisque l'émission/réception d'emails est possible partout, à tout moment. Albert souligne un nouveau paradoxe. Celui du Smartphone, dont il fut initialement demandeur, qui le rassurait dans son organisation mais qui le maintient actuellement dans un cercle vicieux de connexion toujours plus importante :

«A la base, j'étais demandeur d'un BlackBerry, c'est ça le pire ! au début, c'était une aide pour les transports, les avions, le train... je me disais : ça va m'éviter l'effet d'accumulation des mails après une période d'absence, ça va me permettre de me resynchroniser (...) mais très vite, ça a été une spirale où j'ai mon portable, dans la main, je regarde les mails, j'en ai un, j’y réponds, vite, je me décide vite (...)».

\subsubsection{Travail de gestion des urgences et temps de réflexion empêché}

Pour ces 4 cadres, l'email est vecteur d'urgences qu'il faut rapidement prioriser. Ces emails ralentissent puis déconstruisent le travail et l'organisation prévue. Fabien en journée d'observation décrit :

«ça me saoule putain, toujours à trier ces mails, les trucs urgents et les trucs pour hier, pffff : : : (...) le mail est mon ennemi, ça me rajoute des urgences des trucs, des exigences encore que j'avais pas prévu dans mon planning de la journée ».

Albert témoigne :

« les emails au fur et à mesure qu'ils tombent, me font clasher mon organisation, les priorités s'accumulent et moi, je deviens défaillant parce que je maitrise pas

l'arrivée ou le délai d'une action, ça m'agace ».

Anna précise que tous les acteurs de l'entreprise participent à ce contexte d'urgence conduisant à l'usure : 
«chacun vient, m'en met un petit peu dans la tête euh:: le patron fait pareil il arrive un moment, l'éponge elle peut plus quoi ».

Ils empêchent aussi le travail de réflexion :

«ça anéantit mes périodes de réflexion, j'ai plus le temps de prendre du recul sur mes décisions stratégiques et ça c'est nul, je regrette» (Albert).

\subsubsection{Stratégies mises en place pour limiter la surcharge d'emails}

41 Dans certains contextes, les 4 cadres étudiés emploient des stratégies pour tenter de se préserver de la surcharge d'emails. Les solutions ne sont jamais radicales et visent à limiter la surcharge plutôt qu'à la prévenir. Anna décide par exemple d'emporter son ordinateur portable à domicile pour finaliser le traitement de ses emails mais volontairement elle ne prend pas sa prise secteur, pour être limitée par l'autonomie de sa batterie. Elle refuse également de commander un Smartphone pour ne pas subir les effets de «laisse électronique» démontrés plus haut. Certains weekends, Albert s'autorise à éteindre son Smartphone pour ne pas être tenté par ses emails mais il prévient alors ses collègues de sa déconnexion. Géraldine et Anna développent un sentiment de méfiance à l'égard des jeunes générations, qu'elles dénoncent comme responsables de l'accélération de leur surcharge en emails. Elles déplorent leur recours généralisé et peu soigné à l'email, leur participation à des challenges de celui qui se montrera le plus disponible ou le plus engagé en répondant ou en envoyant un email tard dans la nuit. En retour, elles disent accorder moins d'importance au traitement de leurs emails. Les décalages se ressentent et s'expriment dans la qualité du travail collectif.

Ces cadres surchargés par leurs emails se trouvent au cœur d'une injonction paradoxale : celle de vouloir faire coïncider le temps du média - extrêmement rapide - et le temps de la communication interpersonnelle et des actions exigées - extrêmement long (Wolton, 2009). L'outil s'en voit transformé. Pour limiter progressivement leur surcharge, les cadres utilisent l'email de manière quasi-synchrone. La gestion se fait ensuite sur les différents modes de lecture, classement et rédaction d'email qui doivent convenir aux normes et valeurs de chacun.

\subsection{Procédures de lecture, classement, rédaction et exigences implicites}

43 Au-delà du nombre important d'emails que les cadres doivent traiter quotidiennement, les phases de lecture, classement et rédaction exigent une attention et un engagement particuliers. Les 4 personnes étudiées ici permettent de souligner les caractéristiques de tout ce travail de communication médiée, encore peu renseigné dans la littérature.

\subsubsection{Lecture des emails : une activité en deux temps, au pouvoir de distraction important}

Nous l'avons vu, la gestion des emails est continue chez ces 4 cadres. Aussitôt reçu et remarqué sur la messagerie, l'email est traité « au fil de l'eau », selon un premier niveau de lecture nommé « L1 », qui permet aux cadres de se renseigner du contenu global et du niveau d'urgence de la réponse/mission attendue. Pendant cette lecture, 2 cadres s'aident d'outils de priorisation pour faciliter la priorisation des messages parcourus. Albert marque d'un drapeau rouge les emails qui nécessitent une réponse ou une réflexion rapide. Mais sa stratégie l'insatisfait, car à la fin de la journée, il retrouve tous ses emails 
entrants dotés d'un drapeau rouge et est obligé de reprendre la lecture de ces emails. Géraldine pose des filtres automatiques qui colorent ses emails selon le niveau hiérarchique des expéditeurs. Son premier niveau de lecture est facilité mais pas raccourci puisqu'elle s'applique toujours à contrôler la fiabilité des filtres.

C'est seulement dans un second temps, après un niveau de lecture plus approfondi, dit «L2", que les cadres peuvent choisir le traitement à accorder à l'email (classement, formulation d'une réponse ou suppression de l'email). Le délai entre les niveaux L1 et L2 varie en fonction du type d'information reçue. Conserver un email en arrière-plan alourdit la charge cognitive des cadres. Les 4 cadres observés préfèrent alors procéder au niveau de lecture L1 de la première dizaine d'emails entrants, pour ensuite, reprendre les emails jugés prioritaires et entamer la lecture de niveau L2. Des efforts supplémentaires sont nécessaires en niveau de lecture «L2 » lorsque les emails s'avèrent compliqués et délicats. Un seul niveau de lecture suffit parfois pour les emails « faciles, de vie courante, d'un collègue proche ». Cette hétérogénéité des contenus conduit les concepteurs à penser à des solutions de tchat instantané pour faciliter les communications plus spontanées sur des informations pratiques de coordination entre collègues proches ou de renseignement sur sa disponibilité en temps réel. Les outils d'aide à la priorisation et au passage du niveau L1 au niveau L2 tels que les tags, drapeaux, couleurs, police en gras sont aussi à l'étude. Cependant, les questionnaires ont montré que ces outils ne sont pas systématiquement utilisés par les cadres interrogés. À la question : «Utilisez-vous l'une des différentes marques de priorisation (tags, drapeaux, gras, couleurs...) pour gérer vos emails professionnels ?», 24,2\% des 86 cadres ont répondu « Oui » et 74,8\% « Non ». Différentes raisons font que ces cadres n'utilisent pas les marques de priorisation : $43 \%$ ressentent un " sentiment d'inefficacité » de ces outils ; $16,4 \%$ des cadres disent " manquer de temps »; $11,6 \%$ ont des « difficultés à prioriser » les emails reçus et enfin 3,8\% ne les utilisent pas pour d'autres raisons non développées. L'utilisation de ces outils est corrélée au sentiment de surcharge du cadre : plus le sentiment de surcharge du cadre questionné est élevé et moins il utilisera les marques de priorisation $(F(10,344)=2,1594$ et $p=, 01984$; test Lambda de Wilk).

Les cadres subissent aussi le pouvoir d'attraction et de distraction de l'email, les alertes visuelles et sonores sont activées pour chacun d'eux. Anna décrit :

«comme j'ai des alertes qui tombent, j'arrive à le lire en tout petit [l'objet de l'email] ou je reconnais l'expéditeur, voilà bon je vais regarder, et là de fil en aiguille, je vérifie un truc, puis un autre et paf : : je me retrouve à gérer un truc qui est différent du dossier que j'avais pris le matin donc ça, ça, c'est pas bien (...) ça m'ennuie, ça m'agace !».

Mark et al. (2005) ont montré que l'arrivée d'un email, par les niveaux de lecture occasionnés, crée une distraction qui augmente considérablement le temps de retour à l'activité initiale, désorganise et impose un rythme non-prévu qui augmente le temps total nécessaire à la réalisation d'une tâche complète.

\subsubsection{Classement des emails : chronologique plutôt que thématique, avec nécessité de tout conserver}

Après la phase de lecture et de traitement du contenu des emails, les cadres décident d'archiver les communications. L'éclatement des organisations et des missions attribuées à chacun, le nombre d'informations transitant par messagerie, la multiplication des interlocuteurs, associés au manque de temps et à une surcharge d'emails, rendent difficile 
le classement par thématique initialement mis en place par les 4 cadres suivis. Albert raconte: "je ne pouvais plus suivre, toutes les missions se chevauchaient, c'était trop difficile de classer puis ensuite de remettre la main dessus». En effet, pour plus de simplicité et de rapidité dans leur classement d'emails, les 4 cadres surchargés optent désormais pour un classement chronologique, c'est-à-dire qu'ils laissent s'empiler les emails entrants les uns après les autres, et les classent dans le dossier hebdomadaire, mensuel ou annuel correspondant. La fonction « Recherche » des messageries s'avère alors particulièrement salvatrice. Les résultats du questionnaire montrent que, plus les cadres s'estiment surchargés, plus ils utilisent un mode de classement chronologique $(F(3,195)=3,4184$ et $\mathrm{p}=01840$ ). Nos 4 cadres observés ont tous une boîte de réception très chargée (parfois jusqu'à 700 emails non lus), car ils conservent la majorité des emails reçus par mesure de précaution ; cela est d'autant plus vrai que la moitié des cadres suivis travaillent avec des collaborateurs à l'international et préfèrent garder une trace de leurs échanges. Cependant, certains se retrouvent parfois dépassés par ce flux d'informations à gérer puis à stocker. Les capacités de stockage des messageries ne suivant pas la croissance exponentielle des flux d'emails entrants, les cadres doivent régulièrement archiver leurs emails, étape que beaucoup estiment particulièrement lourde et pénible puisqu'elle ralentit tout le système. Les résultats du questionnaire confirment ce point, la deuxième amélioration souhaitée par les cadres serait l'augmentation de la taille des inbox ou serveurs actuels.

\subsubsection{Rédaction des emails : de la prudence avec brouillons à une absence de formes}

48 La phase de rédaction d'un email s'avère délicate et engageante pour les 4 cadres interrogés qui ont conscience de la traçabilité et du transfert potentiellement infini des messages en ligne. Anna témoigne de l'avertissement qu'elle a reçu dès sa prise de poste :

«on m'a clairement dit quand j'suis arrivée : tout document pourrait être utilisé contre vous, donc je ne peux pas faire n'importe quoi, et encore une fois cette partie-là du mail c'est ce qui correspond à ce qu'on faisait dans le temps, quand on dictait y'avait 50 personnes qui relisaient, avec $\mathrm{X}$ signatures, etc. donc cette partie du travail effectivement nécessite un peu plus de réflexion y compris dans l'établissement de la liste de destinataires, parce que maintenant plus personne relit pour moi !».

En fonction du contenu du message à formuler, des enjeux, des destinataires concernés, de l'historique des échanges précédents, la rédaction de l'email est un travail plus ou moins coûteux cognitivement. Pour chaque cadre, il s'agit de se faire comprendre par le destinataire, d'ajuster le message à ce qui est attendu. La difficulté réside dans le fait qu'aucun accord ne fixe les règles d'un email «acceptable». Ce travail de négociation implicite est d'autant plus difficile que les cadres disent manquer de temps de concentration et de réflexion pour la rédaction de leurs emails sur le temps de travail. Pour se lancer dans la rédaction d'un email, 2 des 4 cadres observés rédigent systématiquement un brouillon avant l'envoi d'un message à un supérieur hiérarchique. C'est le cas de Géraldine, qui fait également relire son brouillon à un collègue complice avant de le transmettre au destinataire final :

"ça m'est déjà arrivé plusieurs fois, une personne en qui j'ai parfaitement confiance, la dernière fois, j'ai reçu un mail incendiaire, je commençais d'y répondre et j'ai mis de côté en brouillon et je lui avais demandé son avis, elle amène des éléments et elle m'avait dit 'bah tu t'entêtes, tu sur-interprètes cette chose-là', 
c'est toujours bien dans les périodes de conflit d'avoir d'autres vues parce que souvent j'interprète mal aussi ».

Cette précaution s'est développée après un épisode traumatisant d'échanges d'emails incendiaires avec son patron :

«tu reçois un message pour lequel tu te dis 'merde il se fout de moi !' et là tu réponds immédiatement! Donc émission-réception, tu m’engueules-je t’engueule en gros hein (...) et ben maintenant je mets ça en brouillon et puis je reprends le message le lendemain, donc la plupart du temps je ne l'envoie pas hein, et je le reformule bon, parce qu'on travaille tous en émission-réception donc je me sens agressé bah j'agresse y'a pas de miracle... ».

Ce brouillon est un moyen de faire face à la situation vécue comme stressante et compliquée. Pour structurer sa pensée, mettre ses premières idées à plat, déverser ses premières émotions négatives, le brouillon permet aux cadres de se distancier de l'émotion négative ressentie et de reprendre l'email ultérieurement. Anna souligne ensuite le cas particulier des emails à formuler en anglais notamment :

« il faut faire attention à la façon dont on le libelle, moi j'ai trop vu des industriels avoir des poursuites (...) on considérait que la façon dont les gens parlaient des événements était tout à fait non professionnelle et donc manquement à l'éthique, aux engagements vis-à-vis des autorités, aux engagements vis-à-vis des clients, moi tout ça, ça me fait peur, non là y'a vraiment des règles de communication à mettre en place ».

Les 2 cadres techniques interrogés en entretien d'auto-confrontation ont pris conscience, une fois confrontés aux traces de leurs activités de production d'email, qu'ils cherchaient sans cesse l'exhaustivité de leur propos, qu'ils soignaient au maximum le contenu et la forme des emails envoyés et qu'ils étaient particulièrement prudents sur le choix des mots employés. Colère et énervement sont alors apparus au visionnage de leur activité, car souvent, selon eux, l'heure passée à rédiger un email n'est pas suffisamment « reconnue » par l'interlocuteur, la réponse qui en découle peut d'ailleurs ne pas être à la hauteur.

Parmi les 4 cadres, des exceptions dans l'exigence accordée à ce processus de rédaction d'emails sont exprimées par Albert et Fabien. En entretien, après avoir lu à voix haute un échange d'emails entre lui et une collaboratrice, Albert prend conscience de son style un peu trop rapide: "style rédactionnel, on ne peut plus classique (...) bah ouais, c'est bref quoi. C'est vrai qu'j'suis un peu dur là. Aïe». Fabien rédige également des emails courts mais il n'émet pas de regret par rapport à ce mode de rédaction :

«moi je mets jamais de formule rien, c'est court et basta : Guilaine, je lui écris mon truc et ensuite cordialement (lit l'un de ses emails) voilà, ça c'est mon grand max, moi j'ai pas le temps pour les formes, et j'pense que ça suffit, y'a l'idée principale du mail donc c'est bon ".

Ces deux cadres reçoivent majoritairement des emails soignés et ne reçoivent pas de retour négatif concernant le style de leurs propres emails. Géraldine enfin, quand elle ressent un décalage négatif entre la qualité de la forme reçue et celle qu'elle considère comme "respectueuse ", diminue ses efforts en fonction du respect démontré de son interlocuteur :

«quand je reçois un message bourré de fautes d'orthographe déjà ça a le don de m'énerver donc je ne vais pas m'appliquer autant à répondre correctement parce que je vois pas pourquoi moi je ferais l'effort dans l'autre sens ».

L'observation de cadres très prudents et de cadres moins attentifs à leur rédaction d'email, témoigne de l'absence de règles partagées concernant les critères de forme ou de style attendus de l'email. Il arrive alors que dans la communication interpersonnelle, 
certains s'estiment « lésés, menacés » par un échange, tandis que d'autres ne remarquent pas ou ne prêtent pas attention à leur "menace » écrite. Les cadres ne sont pas formés aux particularités de cette communication médiée. Cette absence de convention ne permet pas de réduire les « décalages » de style où chacun se montre potentiellement de bonne foi. C'est d'ailleurs l'une des raisons à l'origine de l'émergence de ressentis d'« emails problématiques » que nous détaillons ci-dessous.

\subsection{Le fardeau des emails problématiques}

51 L'activité de gestion des emails se complexifie à cause de la réception d'emails jugés «problématiques» par les cadres surchargés étudiés. Aucune catégorisation d'emails problématiques n'a été préalablement imposée aux cadres, ces derniers ont défini, chacun à leur manière, les emails qui leur posaient problème, qui leur semblaient "délicats ", " prise de tête ", " complexes " à gérer. À partir des récits des cadres interrogés, nous avons identifié 5 types d'emails problématiques et repéré les vécus subjectifs associés. Ces emails sont très rarement évoqués dans la littérature et pourtant, les résultats du questionnaire montrent que plus le nombre d'emails gérés augmente et plus le nombre d'emails problématiques reçus augmente $(F(3,196)=5,1920$ et $p=08096)$; cela signifie donc que les cadres surchargés suivis pour cette étude sont davantage exposés à une gestion d'emails problématiques. Les 4 cadres étudiés évoquent tous 5 types d'emails problématiques. Nous détaillons ci-dessous les emails problématiques les plus fréquemment rencontrés et les vécus subjectifs associés. Nous synthétisons ces résultats d'emails problématiques et d'émotions négatives révélées dans le panel 1 et le panel 2.

\subsubsection{Emails problématiques de type « inadéquation du contenu »}

Anna gère fréquemment des emails dont le « contenu est inadéquat ", elle nous en livre un exemple :

« la semaine dernière pendant trois jours on a eu une crise à gérer, j'ai dû recevoir je sais plus 50 ou 60 méga de mails dans la journée, mais ils étaient inutiles parce que la personne s'est lâchée et a envoyé des tas de documents qui servent à rien, en attendant pendant trois jours on avait la boîte bloquée (...) et la personne en face je veux dire elle a pas deux sous de neurones quoi c'est épouvantable ! En plus le doc était pas filtré c'était en allemand donc de toutes les façons y'avait qu'un dixième de la population concernée qui pouvait lire, crrrrrr..., j'ai un peu autre chose à faire !».

Les autres emails problématiques de ce type sont liés à une erreur d'adressage, qui mène souvent à une surcharge informationnelle mal vécue.

Pour les 9 cadres surchargés, cet email problématique « au contenu inadéquat » est celui qui revient le plus fréquemment. Ce sont des emails contenant une information qui n'aurait pas dû être, selon les cadres, véhiculée par email mais plutôt par téléphone ou de visu. Les entretiens avec phases d'explicitation nous permettent de relever différentes expressions émotionnelles: l'énervement est verbalisé par 5 des 9 cadres, car cette inadéquation du contenu implique de nombreuses relectures, complique les relations de communication avec les destinataires. La pénibilité liée au sentiment de perte de temps à la réception de ce genre d'emails est verbalisée par 3 des 9 cadres. Dans ce type de situation, deux cadres changent de canal de communication. $37 \%$ des 86 cadres interrogés par questionnaire indiquent gérer fréquemment des emails au «contenu 
inadéquat ", ce qui en fait le troisième type d'email problématique le plus fréquemment cité.

\subsubsection{Emails problématiques de type « effort d'interprétation à fournir »}

être clair, précis, parce qu'on pouvait pas retourner une lettre aussitôt, maintenant, les collègues, alors je vais pas les nommer hein, mais c'est écrit vite fait et très souvent je comprends pas la moitié de leur pensée ».

Lors de ses échanges avec la Chine ou les États-Unis, le problème de compréhension est handicapant en raison du décalage horaire :

« quand c'est en Chine ou aux US et que les autres dorment et que nous on dormira quand ils seront réveillés, alors j'ai intérêt de comprendre du premier coup ! j'ai pas le choix... Mais bon, la plupart du temps, je m'étonne, alors j'suis obligée de renvoyer : j'ai pas bien compris est-ce que vous pouvez rephraser? (...) c'est terrible et ça m'épuise ».

Lorsqu'un échange se complique, au-delà de 5 emails d'incompréhension, Anna privilégie alors le contact téléphonique. Cela renvoie à un email problématique de type « effort d'interprétation ", également géré par tous les cadres du panel 1. Pour comprendre et parfois même déchiffrer l'email reçu, les cadres surchargés sont contraints de produire des efforts d'interprétation, assez coûteux en termes de temps passé à lire puis relire les emails. Ce sont les deuxièmes types d'emails estimés « problématiques » chez les 9 cadres surchargés ( 8 déclarent en recevoir très régulièrement) et chez les 86 cadres du panel 2 ( $43 \%$ déclarent en recevoir très régulièrement). Cet effort de compréhension est encore plus important quand l'email reçu est rédigé en anglais. Le niveau de surcharge des cadres est tel que, prendre du temps pour comprendre un email engendre de l'énervement et du stress pour le tiers des 9 cadres surchargés. Pour faire face à ces émotions négatives, certains utilisent le téléphone, notamment quand le sujet évoqué est complexe, tandis que d'autres se désengagent de la conversation par email.

\subsubsection{Emails problématiques de type » travail supplémentaire à fournir dans l'urgence "}

Albert déplore ensuite les « comportements débiles comme le type qui m'envoie une pièce jointe avec un doc de 100 pages, 2 minutes avant la réunion en disant en début de réunion: 'bon alors j'espère que vous avez lu dans les grandes lignes ce que je vous ai envoyé tout à l'heure !' Ah non mais le type ! C'est absurde, et ça m'énerve profondément, on en vient à des trucs irréalistes, sans la messagerie, le type n'aurait jamais osé faire ça! ». Ces emails du type "travail supplémentaire à fournir dans l'urgence », désignent principalement des fichiers Power Point ou de longs documents Word envoyés en dernière minute par l'interlocuteur et impossibles à lire dans le temps imparti. Ces emails problématiques sont rencontrés par 6 des 9 cadres observés. Les émotions verbalisées en entretiens avec phases d'explicitation sont : l'énervement, l'agacement et la méfiance due à la crainte par anticipation des emails entrants qui contiendront du travail à réaliser urgemment (pour 4 d'entre eux). Pour faire face à ces exigences de travail, certains cadres laissent passer l'email et n'y répondent pas, d'autres réalisent la mission confiée mais dans un temps plus court, avec une précision d'analyse de moindre qualité. D'autres encore, travaillent en dehors des heures de travail pour parvenir à faire le travail 
demandé dans le laps de temps ordonné. $66 \%$ des 86 cadres surchargés interrogés disent gérer ce type d'emails, ce qui en fait l'email problématique le plus fréquemment cité.

\subsubsection{Emails problématiques de type « attaque et agressivité »}

\section{principalement les emails véhiculant "attaque et agressivité », provoqués notamment par} ses jeunes collègues managers :

« de jeunes responsables de groupe qui ne savent pas manager leurs troupes, qui ne savent pas dire entre 4 yeux aux personnes ben t'as mal bossé, ça, ça va pas (...) j'vois souvent tomber un ordre qui arrive du haut, il arrive en bas déformé parce que le téléphone arabe c'est un truc du feu de dieu, c'est forcément déformé à chaque endroit du chaînon, en l'occurrence du mail ! Avec la terre entière en copie ! Je le vis très mal, c'est insupportable ! Parce que moi dans ces cas-là, je me sens attaquée, je sais pas trop comment me comporter vis-à-vis du manager parce qu'en plus, y'a des témoins, ça reste tracé, et le risque c'est qu'on me le ressorte ! ».

Aussi, Géraldine raconte le cas particulier d'un collègue qu'elle identifie comme « danger public » dans ses productions d'emails :

« Arthur (...) par messagerie il m'écrivait des horreurs! Avec copie à plein de gens ! (...) alors bon, je le connaissais un peu, alors quand je recevais un de ses mails, je me posais 5 minutes, je soufflais, et je cherchais ce qu'il voulait me dire au-delà du truc sec, des mots agressifs, des majuscules, des fautes, des reproches... C'était de la perte de temps de se creuser la tête en se disant mais voyons voir : qu'est-ce qu'il me demande réellement? Qu'est-ce que ça sous-entend? Pourquoi il me le demande de cette façon-là ?».

Gauducheau (2012) est la seule à avoir identifié ce type d'emails comme étant difficile à gérer pour les utilisateurs et surtout source d'émotions négatives. 6 des 9 cadres évoquent ce problème d'email porteur d'agressivité; les mots "secs", les phrases courtes, les brimades de mise en accusation avec témoins, la multiplication des signes d'exclamation sont ressentis comme agressifs par les récepteurs qui expriment alors de l'énervement, de la colère, de la menace à la lecture de ceux-ci (2 des 9 cadres). Pour y faire face, les cadres mettent en place diverses stratégies. Géraldine rencontre sans hésiter les différents protagonistes expéditeurs dudit email agressif, un autre cadre privilégie la rencontre et le contact en face à face, ou au minima téléphonique, ou se désengage totalement de l'échange. $26 \%$ des 86 cadres surchargés disent gérer ce type d'emails problématiques. C'est le quatrième type d'email problématique le plus fréquemment géré. Le statut de cadre, commun aux individus constitutifs de nos deux panels, permet peut-être d'expliquer cette faible proportion recensée. On peut faire l'hypothèse que les cadres sélectionnés pour l'étude ont de hauts niveaux de responsabilité, qui leur confèrent un minimum de protection face à ces agressions de subordonnés, cependant, lorsqu'un email de ce type est reçu, l'intensité des émotions négatives exprimées est importante.

\subsubsection{Emails problématiques de type « inadéquation de la forme »}

Anna gère aussi des emails problématiques de type «inadéquation de la forme » et en explique le contenu :

« En fait regardez, les messages, très souvent le bonjour n'y est pas, le s'il vous plaît n'existe plus et quant au merci : en rêve ! ! Donc quand on lit les messages c'est de l'agression perpétuelle, pas forcément consciente, mais de l'agression perpétuelle 
(...) dans mon coin, j'peux pas m'empêcher de pousser une grande bordée de jurons

et bon de dire bon sang vous avez pas fini vos âneries! ».

Dans notre étude, 4 des cadres surchargés sont particulièrement attentifs à la forme des emails reçus, à savoir les formules de politesse, l'orthographe, la présentation du texte, le mode rédactionnel et la syntaxe. Les entretiens avec phases d'explicitation révèlent surtout de la colère, de l'énervement et de l'agacement ( 3 d'entre eux), car l'inadéquation de la forme des emails reçus est perçue comme une offense, un manque de respect de l'expéditeur pour le destinataire. $13 \%$ des 86 cadres interrogés sont concernés par cet email problématique.

\subsubsection{Synthèse des emails problématiques et des émotions négatives engendrées}

La figure 2 présente les 5 types d'emails problématiques et les émotions négatives associées les plus fréquemment exprimés par les deux panels de cadres surchargés étudiés. Les quantifications sont directement extraites des entretiens (pour le panel 1) et des questionnaires (pour le panel 2). Les émotions négatives n'ont pas été recueillies par questionnaire, car nous avons considéré difficile et peu fiable de les recueillir sans resituer l'individu à un moment vécu particulier.

Figure 3 : Types d'emails problématiques gérés par les panels 1 et 2 de cadres surchargés et émotions négatives engendrées.

Figure 3: Types of problematic email managed by the panels 1 and 2 of overloaded managers, and the negative emotions caused

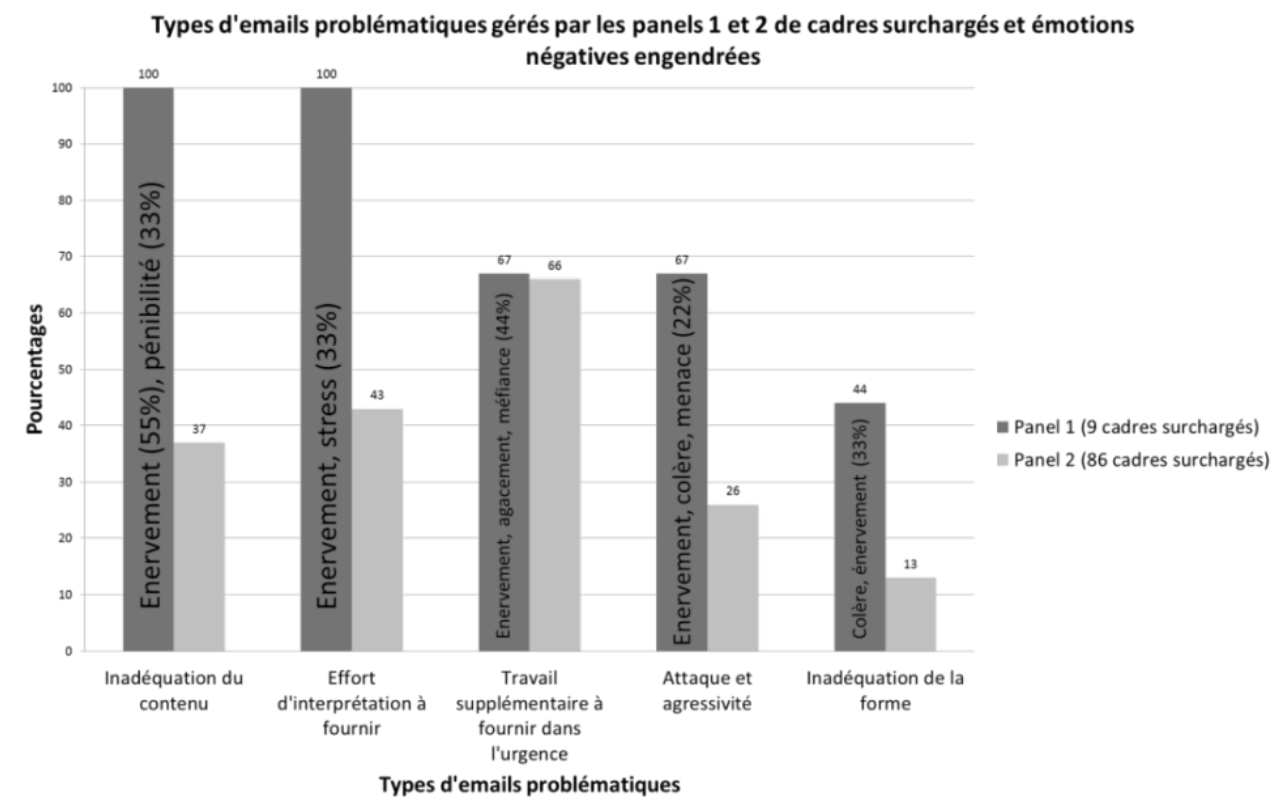

60 Les différences de perception observées entre les deux panels peuvent avoir plusieurs explications possibles : (1) la méthodologie de recueil de données inspirée des entretiens d'explicitation permet aux cadres de prendre conscience et d'expliciter certains cas précis d'emails problématiques, alors que le questionnaire distribué hors-contexte demande aux cadres de se reconnaitre dans les catégories prédéfinies, sans aide ou guidage pour se remémorer les emails et les situations vécues comme problématiques. De ce fait, il y a peut-être eu pour le panel 2 une «sous-estimation" des emails problématiques. (2) les 86 cadres surchargés du panel 2 étaient peut-être moins 
surchargés que nos 9 cadres qui se sont portés volontaires pour l'étude tant cette surcharge leur pesait.

61 Après avoir exposé les difficultés liées à la gestion des emails, aux procédures de lecture/ classement/rédaction et aux emails problématiques, nous proposons quelques pistes d'amélioration.

\subsection{Pistes socio-techniques d'amélioration}

\subsubsection{Pistes d'amélioration organisationnelles}

La messagerie électronique s'est insidieusement imposée aux cadres comme le canal de communication principal pour leurs échanges professionnels depuis désormais plus de 10 ans, les habitudes individuelles et collectives se sont sédimentées dans les organisations, sans réel consensus ni réelle discussion concernant les règles et normes d'usage. La discussion de recommandations organisationnelles semble donc prioritaire.

L'amélioration des usages de l'email doit impliquer toutes les strates de l'organisation, qui doit reconnaitre l'importance et les effets potentiellement dramatiques d'une surcharge d'emails sur le travail, le collectif de travail et le bien-être de l'individu au travail. Plusieurs aménagements et améliorations sont testés dans les entreprises et institutions pour atténuer les effets néfastes de la messagerie (chartes, guides, formations, nouvelles règles...). Cependant, les 9 cadres étudiés n'ont jamais trouvé satisfaction dans les améliorations proposées par leurs organisations: ils jugent les formations inadaptées, ennuyeuses et difficiles à concilier avec leur surcharge quotidienne ; et estiment les chartes de bon usage de la messagerie trop vagues dans leurs recommandations ou remplies de conseils de bon sens. Les résultats issus du questionnaire sont plus modérés, $39 \%$ des 86 cadres surchargés se disent intéressés par des formations d'accompagnement à la gestion de leur messagerie.

Nous promouvons plutôt des séances de retours d'expériences entre participants volontaires souhaitant échanger sur les stratégies de coping disponibles pour faire face à leurs surcharges d'emails et pour communiquer autour de leurs différentes attentes du média. L'idée serait d'aboutir à la fin de ce REX à un guide collaboratif renseignant les attentes concernant le style et le contenu d'un email jugé « acceptable ». S'il est difficile de prescrire un modèle de l'email idéal, il est possible de donner des exemples d'emails jugés "problématiques" par chacun. Comme le soulignent Bobillier-Chaumon et Triposelli (2012), c'est lorsqu'un espace de délibération se créé autour de l'outil que la pratique deviendrait «bonne» et acceptée, en changeant le regard sur l'outil, qui ne devient plus source de tension et de surcharge mais plutôt aide dans l'amélioration des capabilités de communication dans le travail. La discussion prend du temps et nécessite l'implication de toutes les parties de l'entreprise. Sans consensus, il devient difficile de croire en la pérennité du changement. Des formations personnalisées peuvent ensuite permettre aux cadres d'utiliser les outils les plus récents ainsi que toutes leurs fonctionnalités associées pour se faciliter la gestion quotidienne d'emails.

La tenue de ces REX et de ces formations soulève toutefois 2 questions : la première relève de la difficulté pour un opérateur déjà surchargé d'accepter de participer à ces moments réflexifs au détriment de leur travail; la seconde relève du fait d'oser se montrer "défaillant» dans sa gestion des emails aux yeux de collègues ou de supérieurs hiérarchiques (Géraldine imagine une dérive possible: les participants à ces formations 
peuvent constituer, aux yeux d'une organisation peu empathique, les opérateurs les moins "armés" pour évoluer par exemple). Enfin, à l'issue de ces formations, l'organisation peut prétendre exiger toujours plus de travail et de communication à l'opérateur, désormais " équipé " pour faire face à cette surcharge. Ces formations sont donc également un excellent moyen de déculpabiliser l'organisation du travail, responsables de l'intensification des rythmes de travail et des risques psycho-sociaux qui s'en suivent.

\subsubsection{Pistes d'amélioration techniques}

Plusieurs améliorations d'ordre technique peuvent être envisagées pour améliorer la gestion des emails chez les cadres surchargés. Elles semblent plus aisées à mettre en place que les recommandations organisationnelles présentées précédemment, mais leurs retombées finales sur l'activité problématique étudiée seront probablement moins importantes.

Tout au long de nos observations, nous avons pu observer les usages sur deux logiciels distincts de messagerie ${ }^{15}$. Le premier, largement utilisé dans les organisations de travail, manque de transparence (d'affordance) pour l'utilisation des marques de priorisation et des arborescences de classement qui ne se distinguent pas assez de l'arborescence du disque dur. Chez certains cadres, l'email déjà lu reste en gras, ce qui oblige l'utilisateur à ré-ouvrir l'email pour savoir s'il l'a déjà traité ou non (double traitement de l'information et perte de temps). Le manque de simplicité au niveau des insertions des pièces jointes pose problème pour certains cadres, il serait préférable de permettre de "glisser » des pièces jointes depuis le bureau jusque l'email. Certains utilisateurs se plaignent de l'utilisation de la fonction " répondre à tous ", ainsi pourquoi ne pas songer à une sorte de double validation pour l'utilisation de la dite fonction: lorsque l'on sélectionne " répondre à tous ", un message afficherait : "Êtes-vous sûr de vouloir utiliser cette fonction ?». Les boutons d'activation/désactivation de l'alerte visuelle et sonore devraient être, eux aussi, plus affordants sur l'interface, pour permettre aux utilisateurs de diminuer les phénomènes d'interruptions. Le second logiciel, dans sa nouvelle version (l'apparition de la fenêtre interruptive "vous avez reçu un nouvel email ", caractéristique de la première version, a été éliminée), offre quant à lui de nombreuses fonctionnalités de priorisation, pour jongler entre plusieurs outils de communication (messageries électronique, instantanée, réseaux sociaux, plateformes de partage...). Ce logiciel nécessite un certain temps d'appropriation pour ne pas se perdre dans les dédales d'options.

Le manque de mémoire de la messagerie électronique qui oblige les utilisateurs à lancer des processus d'archivage est un autre point souvent évoqué par les cadres utilisateurs des deux types de logiciels. C'est aussi la seconde amélioration souhaitée par les 200 cadres interrogés via questionnaire. En effet, les processus d'archivage sont très longs et bloquent l'utilisation d'autres logiciels.

À la mise en place de ces diverses pistes de recommandations organisationnelles et techniques, il ne subsiste pas moins de questions concernant l'intensification des rythmes de travail actuels, l'accroissement des communications médiées par les TIC, les collaborations avec des sites éclatés à l'international, la productivité poussée à l'extrême et petit à petit, la compression de l'humain au travail, positionné comme absorbeur de stress, de pression et de charge de travail plutôt que comme «sujet capable » (Pastré, 
Mayen, \& Vergnaud, 2006). La discussion qui suit porte sur les résultats issus de l'étude des 4 cadres surchargés.

\section{Discussion}

70 Cet article visait à comprendre les caractéristiques de l'activité de gestion des emails et les expériences vécues associées de cadres s'estimant surchargés par leurs emails. Plusieurs méthodes ont été mises en place pour parvenir à éclairer différents points de vue de l'activité. Parmi un premier panel de 9 cadres, nous avons sélectionné 4 cadres particulièrement surchargés par leurs emails, aux caractéristiques de gestion et de régulation d'emails différentes. Nous avons successivement décrit les contextes socioprofessionnels de ces cadres, le phénomène de gestion continue des emails leur permettant de tenter de faire face à l'explosion d'emails et à la complexité des échanges, les effets délétères sur leur santé et efficacité au travail. Nous avons souligné les spécificités des phases de lecture/classement/rédaction d'emails, coûteuses cognitivement et émotionnellement pour ces cadres surchargés. L'identification d'emails «problématiques » et les émotions négatives associées sont apparues comme l'une des originalités de l'étude, soulignant à nouveau le manque de repères et de règles communes aux usages hétérogènes du média. Enfin, des recommandations permettent de discuter des perspectives à donner à la messagerie électronique.

71 L'email est un cas particulier des nouveaux outils de communication, il incarne l'une des sources principales et le symbole majeur de «stress au travail » des cadres d'aujourd'hui (Barley et al., 2011). La messagerie électronique, par le contenu incessant d'emails qu'elle amoncelle, incarne pour les cadres connectés, un rappel tangible permanent et anxiogène de leur surcharge. Le décalage intenable réside entre la rapidité du média et la lenteur des communications interpersonnelles, que chacun tente d'absorber et de négocier au mieux pour répondre aux normes sociales de réactivité attendues. Pour maintenir un certain sens du contrôle, les cadres surchargés par leurs emails se résignent à une connexion et à une gestion continue de leur messagerie électronique. Les cadres cherchent à anticiper les problèmes futurs, à se rassurer sur le bon déroulement des actions engagées et à ne pas prendre de retard sur les missions confiées. L'effet de réassurance est de courte durée puisqu'avec cette connexion permanente, la masse d'informations reçues et l'urgence véhiculée, c'est un réservoir d'informations jugées anxiogènes qu'il faut parvenir à traiter malgré tout. Les cadres se disent « prisonniers » de leurs messageries, car si elles leur offrent une visibilité temps réel sur l'activité en train de se faire, elles permettent à l'organisation de vérifier les qualités de disponibilité, réactivité et engagement des cadres sollicités. Cela conduit à des "jeux de pouvoir » et à des "démonstrations de présence déplaisantes" soulignés par deux cadres du panel. Les conséquences de cette gestion continue ont été mises à jour. Pour parvenir à diminuer leur surcharge d'emails, les cadres allongent leur temps de travail, quitte à le terminer à domicile pour bénéficier de périodes calmes propices à la réflexion, jugées «trop rares et diminuées au bureau». À cette porosité de la frontière travail-hors travail correspond l'expression d'émotions négatives telles que l'agacement, l'énervement dont les conséquences d'usure et de fatigue sont explicitement mentionnées. Les entretiens et la proposition d'un journal de bord personnel ont permis aux cadres étudiés de prendre conscience de cette gestion débordante de l'email, de la quantifier, de l'observer avec plus de recul et d'objectivité. Généralement, les usagers sous-estiment leur usage de la 
messagerie (Renaud et al., 2006), ici, ils ont exprimé de la surprise et de la peur à se constater « addicts » à leurs emails. L'effet était d'autant plus marquant pour les cadres qui disposent d'un Smartphone, nouvelle «laisse électronique» qui ajoute un fort pouvoir distracteur sur les cadres, qui consultent alors sans limite spatio-temporelle. La " pause-mail » se substitue à la traditionnelle " pause-cigarette ». Cette gestion continue nécessite un travail complexe de priorisation des urgences, l'organisation et la planification des cadres sont rapidement déconstruites au profit de la nouvelle tâche introduite par l'email. Lorsqu'un cadre répond rapidement, il est tacitement attendu qu'il poursuive ainsi, quel que soit le contexte. Tous ces résultats confirment effectivement que ce n'est pas la technologie qui accélère ou diminue le stress des cadres (par exemple, ce n'est pas le nombre d'emails reçus qui détermine si le cadre se sentira ou non surchargé par ses emails) mais bien les usages qui en sont faits (Duxbury, Towers, Higgins, \& Thomas, 2006), individuellement mais surtout collectivement, qui sont ensuite capables d'impacter fortement le travail et le vécu subjectif des cadres.

72 À la suite de Gauducheau (2012) qui identifiait les émotions négatives engendrées par certaines phases de traitements des emails ${ }^{16}$, nous sommes parvenus à détailler le déploiement de cette activité engageante de gestion d'emails et les émotions associées depuis la lecture jusqu'à la rédaction d'un email, en passant par l'étape de classement. Dès la phase de lecture, l'emprise de l'email sur les cadres est telle qu'ils se disent agacés puis tristes que ce média puisse dicter le rythme de leur vie professionnelle et personnelle. L'importance accordée à la phase de classement des emails souligne la méfiance déployée à l'égard des messages, qui constituent désormais des preuves en cas de litige interne ou externe à l'entreprise. La phase de rédaction d'email enfin, estimée délicate par la majorité des cadres observés (tous panels confondus), laisse chacun organiser le contenu et la forme de son message selon les critères qu'il considère comme acceptable. La prudence nécessaire à cette rédaction est chronophage et certains cadres s'énervent de constater le temps " perdu » à la recherche de formulations adéquates, alors que d'autres se découvrent trop secs et lapidaires. Les difficultés de ce média résident dans le fait que les utilisateurs n'ont pas tous conscience de l'hétérogénéité de leurs pratiques et modes de gestion des emails, et qu'il n'a jamais existé de normes ou de règles collectives visant à minimiser les effets douloureux de cette hétérogénéité. La gestion des emails est ensuite compliquée par l'arrivée d'emails dits «problématiques». Les entretiens et journaux de bord nous ont permis d'identifier 5 types d'emails "problématiques» du fait de leur contenu/forme inadapté, de leur caractère agressif/menaçant, ou du travail supplémentaire qu'ils exigent (inintelligibilité du message ou travail à fournir dans l'urgence). À notre connaissance, la littérature ne souligne pas l'existence de tels messages, pourtant ils apparaissent comme un fardeau supplémentaire pour ces cadres, entraînant une kyrielle d'émotions négatives et de la lassitude à long terme. Ils sont dévastateurs pour le collectif puisque les tensions et les conflits restent larvés à travers le média. Selon 3 cadres interrogés du panel 1, l'émergence d'emails problématiques est aussi accentuée par les "jeunes générations et leur recours massif à l'email », quels que soient les motifs du message à échanger, utilisé selon le même mode qu'un message instantané. Les différences générationnelles viennent donc accentuer les manques de repères et flouter encore plus les usages à adopter avec l'email.

73 L'étude de ces cadres particulièrement surchargés par leurs emails, en souffrance par rapport à ce travail en pleine mutation, a nécessité une certaine prudence dans nos analyses mais aussi dans la suite à donner à celles-ci. Les cadres étaient en attente d'une 
aide à la réflexivité, de pistes d'amélioration pour leur gestion quotidienne. Les recommandations proposées ne sont pas exhaustives et nous les avons systématiquement nuancées. Nous pensons en effet que pour réduire les effets pervers de cette «servitude volontaire " (Durand, 2004) à l'email, l'organisation et les différents niveaux de management doivent reconnaître : le travail exigé par ces emails, les émotions négatives engendrées par ce traitement quotidien, l'empêchement du travail réflexif dû aux réponses et aux délais toujours plus courts exigés à travers le média. Pour Bretesché et al. (2012), l'autonomie « réelle » des cadres se mesure désormais dans la « capacité du cadre à pouvoir se déconnecter pour mettre en cohérence des événements pluriels ». Kushlev et Dunn (2015) ont effectivement montré qu'un nombre limité de 3 connexions quotidiennes à la messagerie diminuait le sentiment de débordement par rapport à un nombre illimité de connexions. La déconnexion périodique comme solution de minimisation du débordement est testée par plusieurs entreprises. Malheureusement, les améliorations relevées aujourd'hui sont encore peu probantes (Prost \& Zouinar, 2015). À notre sens, la mise en place et la réussite d'une telle mesure semblent difficiles tant qu'elle n'est pas encouragée par l'organisation tout entière, tant qu'elle n'est pas désignée comme "la » nouvelle norme louable de communication de l'entreprise. Associés à ces aménagements de déconnexions collectives volontaires, il serait pertinent de revenir à des communications et négociations formelles ou informelles en face à face. Comme le souligne Wolton (2009), ces négociations présentielles permettent de résoudre les problèmes d'incommunication, facilitant la cohabitation de tous les points de vue, même contradictoires, et donnant à chacun la possibilité de conserver son identité et ses capabilités.

\section{BIBLIOGRAPHIE}

Assadi, H., \& Denis, J. (2005). Les usages de l'e-mail en entreprise : Efficacité dans le travail ou surcharge informationnelle ? In E. Kessous \& J.-L. Metzger (Eds.), Le travail avec les technologies de l'information (pp. 135-155). Paris : Hermès.

Bardin, L. (1977). L'analyse de contenu. Paris : PUF.

Barley, S. R., Meyerson, D. E., \& Grodal, S. (2011). E-mail as a Source and Symbol of Stress. Organization Science, 22(4), 887-906

Blanc, G., \& Ettighoffer, D. (2002). Pour une écologie du temps. Sociétal, $n^{\circ} 37$.

Bobillier-Chaumon, M.E., \& Triposelli, L. (2012). L'objet technique, possible instrument de développement de l'activité ? De la notion d'usage à une vision métier. In Actes du Congrès International d'Ergonomie, SELF, Lyon, France.

Bobillier-Chaumon, M.E. (2012). L'impact des technologies sur le travail des cadres (Synthèse de la recherche). Université de Lyon - Laboratoire GRePS / APEC.

Bretesché, S., de Corbière, F., \& Geffroy, B. (2012). La messagerie, principale métronome des activités de cadres. Nouvelle Revue du Travail, 1. 
Cahour, B. (2012). Les émotions vécues, constitutives de l'activité. Cas des interactions de travail et des usages situés. Document de synthèse. Habilitation à Diriger les Recherches, Université de Picardie.

Cahour, B., \& Salembier, P. (2012). The user phenomenological experience : Evoking the lived activity with "re-situating" interviews. Paper presented at the Workshop on Theories Behind UX Research and How They are Used in Practice, CHI'2012, May 5-10, Austin, Texas.

Cahour, B., Salembier, P., \& Zouinar, M. (2016, à paraître). Analyser l'expérience vécue de l'activité. Le Travail Humain.

Chesley, N., Moen, P. \& Shore, R.P. (2003). The new technology climate. In P. Moen (Ed.), It's about time : couples and careers (pp. 220-241). Ithaca, NY : Cornell University Press.

Créno, L., \& Cahour, B. (2015). Triangulation des méthodes pour une analyse de l'activité selon différents points de vue : exemple de la gestion des emails chez des cadres surchargés. Psychologie Française, 60(2), 129-144.

Datchary, C., \& Licoppe, C. (2007). La multi-activité et ses appuis : l'exemple de la « présence obstinée » des messages dans l'environnement de travail. Activités 4(1), 4-29.

De Gasparo, S., \& Van Belleghem, L. (2013) L'ergonomie face aux nouveaux troubles du travail : le retour du sujet dans l'intervention. In F. Hubault (Ed.), Persistance et évolutions : les nouveaux contours de l'ergonomie. Actes du séminaire de Paris 1 des 11, 12 et 13 juin 2012. Toulouse : Éditions Octarès.

De la Rupelle, G., Fray, A.M., \& Kalika, M. (2014). Messagerie électronique, facteur de stress dans le cadre de la relation managériale, Revue de gestion des ressources humaines, 2014/1, n 91.

Ducheneaut, N., \& Bellotti, V. (2001). Email as a habit : An exploration of embedded personal information management. Interactions, 8(5), 30-38.

Durand, J.P. (2004). La Chaîne invisible. Travailler aujourd'hui : flux tendu et servitude volontaire. Paris : Le Seuil.

Duxbury, L. E., Towers, I., Higgins, C., \& Thomas, J.A. (2006). From 9 to 5 to 24/7 : How technology has redefined the workday. In W. K. Law (Ed.), Information Resources Management : Global Challenges (pp. 305-332). Hershey, PA : Idea Group Publishing.

Fortin, M.F. (1996). Le processus de la recherche : de la conception à la réalisation. Ville Mont-Royal, Québec : Décarie éditeur inc.

Gauducheau, N. (2012). L'expérience du courriel en situation professionnelle : représentations de l'activité, jugements et affects. Activités, 9(2), 88-111.

Gueguen, G. (1997). L'entreprise doit-elle réagir face aux turbulences environnementales ? Propos de réflexion, 6ème Conférence Internationale de Management Stratégique (AIMS), 24-27 juin 1997, HEC Montréal, Canada, (Actes).

Jauréguiberry, F. (2010). Pratiques soutenables des technologies de communication. Projectics, $n$ ${ }^{\circ} 6,107-120$.

Jauréguiberry, F. (2012). Retour sur les théories du non-usage des technologies de communication. In S. Proulx \& A. Klein (Eds.), Connexions : communication numérique et lien social (pp. 335-350). Namur : Presses universitaires de Namur.

Kalika, M., Boukef Charki, N., \& Isaac, H. (2007). La théorie du millefeuille. De la non-substitution entre communications électroniques et face à face. Revue Française de Gestion, 172, 117-130.

Klein, T., \& Ratier, D. (2012). L'impact des TIC sur les conditions de travail. Rapport du Centre d'analyse stratégique et de la direction générale du travail, 49, 328p. 
Kushlev, K., \& Dunn, E. W. (2015). Checking email less frequently reduces stress. Computers in Human Behavior, 43(C), 220-228.

Lahlou, S. (2000). Attracteurs cognitifs et travail de bureau. Intellectica : revue de l'Association pour la Recherche Cognitive, 30, 75-113.

Lazarus, R., \& Folkman, S. (1984). Stress, appraisal, and coping. New York : Springer.

Lee, J., \& Pantelli, N. (2011). You got email! The cases of inter-organizational collaboration for engineering Product design. Engineering Management Journal, 23(3), 18-21.

Mano, R. S., \& Mesch, G. S. (2010). E-mail characteristics, work performance and distress. Computers in Human Behavior, 26(1), 61-69.

Mark, G., Gonzalez, V.M., \& Harris, J. (2005). No task left behind ? : examining the nature of fragmented work. Proceedings of the SIGCHI Conference on Human Factors in Computing Systems, April 02-07, Portland, Oregon, USA.

Mark, G., Voida, S., \& Cardello, A. (2012). A pace not dictated by electrons : an empirical study of work without e-mail. Proceedings of the 2012 ACM annual conference on Human Factors in Computing Systems, 555-564.

Observatoire sur la Responsabilité Sociétale des Entreprises (ORSE) (2011). Guide de « bon usage de la messagerie électronique dans un cadre professionnel. [En ligne]. Disponible sur : www.orse.org

Pastré, P., Mayen, P., \& Vergnaud, G. (2006). La didactique professionnelle. Revue française de pédagogie, 154, 145-198.

Popma, J. (2013). Technostress et autres revers du travail nomade. Working paper, European Trade Union Institute (ETUI). http://www.etui.org/content/download/12197/101001/file/13+WP+2013 $+07+$ Popma+Tech nostress+FR+Web+version.pdf

Prost, M., \& Zouinar, M. (2015). De l'hyper-connexion à la déconnexion : quand les entreprises tentent de réguler l'usage professionnel des e-mails. Perspectives interdisciplinaires sur le travail et la santé (PISTES), 17-1.

Przybylski, A.K., Murayama, K., DeHaan, C.R., \& Gladwell, V. (2013). Motivational, emotional, and behavioral correlates of fear of missing out. Computers in Human Behavior, 29(4), 1841.

Renaud, K., Ramsay, J., \& Hair, M. (2006). You've Got E-Mail !... Shall I Deal with It Now? Electronic Mail from the Recipient's Perspective. International Journal of Human-Computer Interaction, 21(3), 313-352.

Salembier, P., Theureau, J., \& Relieu, M. (Eds.). (2004). Introduction au numéro spécial « Activité et Action/Cognition Située ». Activités, 1(2), 3-10.

Shenk, D. (1998). Data Smog : surviving in the information glut. San Francisco : Harper, San Francisco.

Suchman, L. A. (1987). Plans and situated actions - The problem of human-machine communication. Cambridge : Cambridge University Press.

Tahri, W., \& Fallery, B. (2010). L'usage de la messagerie électronique : une méta-analyse des travaux francophones sur la période 2000-2008. Management \& avenir, 184-200.

Taylor, H., Fieldman, G., \& Altman, Y. (2008). E-mail at work : a cause for concern ? The implications of the new communication technologies for health, wellbeing and productivity at work. Journal of organisational transformation and social change, 5(2), 159-172. 
Terssac, G. de. (1990). La genèse des savoir-faire. Les savoirs professionnels : Travail, idéologies, pratiques, 9, 133-145.

Theureau, J. (2002). Cours d'expérience, cours d'action, cours d'interaction : essai de précision des objets théoriques d'étude de l'activité individuelle-sociale. $4^{\circ}$ Journées Act'ing “Objets théoriques, objets de conception, objets d'analyse \& situations d'étude privilégiées”, 6-7 Juin, Nouan-Le-Fuzelier.

Théry, L. (2006). Le travail intenable. Résister collectivement à l'intensification du travail. Paris : La Découverte, collection « Entreprise \& Société ».

Vermersch, P. (1994). L'entretien d'explicitation. Les cahiers de Beaumont, 52 bis-53, 63-70.

Whittaker, S., \& Sidner, C. (1996). Email overload : exploring personal information management of email. In Proceedings of the SIGCHI conference on Human factors in computing systems : common ground, Vancouver, British Columbia, Canada, 276-283.

Wolton, D. (2009). Informer n'est pas communiquer. Paris : CNRS Édition, collection Débats.

Zouinar, M., \& Cahour, B. (2013) Activités et expérience vécues : quels liens ? Épique 2013, 10-13 juillet, Bruxelles.

\section{ANNEXES}

\section{Annexe 1}

Exemple d'une grille d'observable utilisée en journée d'observation auprès du panel des 9 cadres surchargés

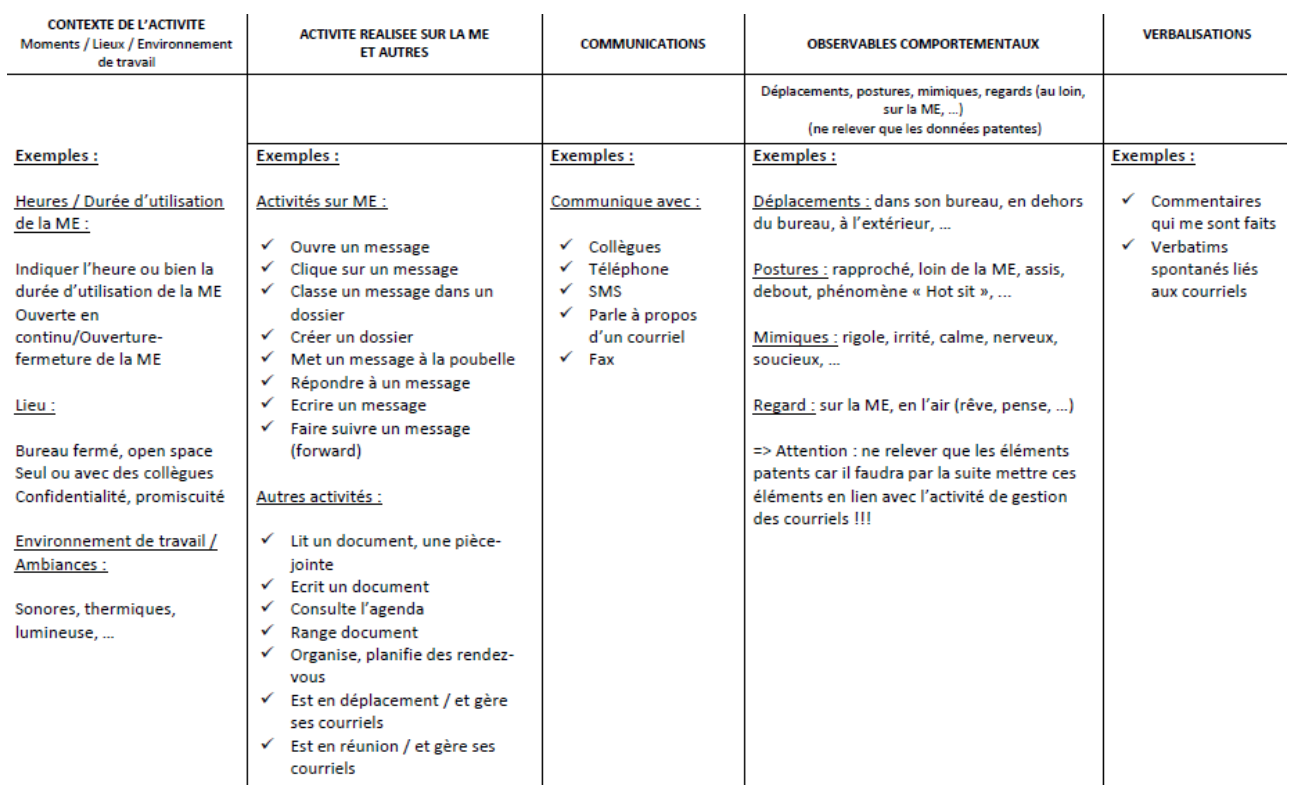


Extrait du journal de bord fourni au panel des 9 cadres surchargés

\begin{tabular}{|c|c|c|c|c|c|c|}
\hline $\begin{array}{c}\text { Heure } \\
\text { d'utilisation } \\
\text { de la } \\
\text { messagerie } \\
\text { (ou durée } \\
\text { d'utilisation) }\end{array}$ & \begin{tabular}{|c|} 
Contexte \\
d'utilisation \\
(à mon poste de \\
travail, en \\
réunion, chez \\
moi, au \\
restaurant,...)
\end{tabular} & $\begin{array}{l}\text { Supports utilisés } \\
\text { (PC, } \\
\text { Smartphone, } \\
\text { Tablette,...) }\end{array}$ & $\begin{array}{c}\text { Nombre } \\
\text { de } \\
\text { nouveaux } \\
\text { courriels } \\
\text { gérés }\end{array}$ & 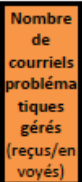 & $\begin{array}{l}\text { Commentaires } \\
\text { (messagerie ouverte en continu/discontinu, explication du courriel problématique, } \\
\text { précision sur ce qui a été fait dans la messagerie : lecture/réponse/...) }\end{array}$ & $\begin{array}{l}\text { Moments } \\
\text { de } \\
\text { surcharge } \\
\text { particuliè } \\
\text { rement } \\
\text { intenses }\end{array}$ \\
\hline \multicolumn{7}{|c|}{ Le ..../.../2012 } \\
\hline & & & $\ldots+\cdots$ & $\ldots$ & 1 & $\ldots \ldots$. \\
\hline & & & & & & \\
\hline & & & & & & \\
\hline & & & & & & \\
\hline & & & & & & \\
\hline & & & & & & \\
\hline & & & & & & \\
\hline & & & & & & \\
\hline & & & & & & \\
\hline
\end{tabular}

\section{Annexe 2. Questionnaire (papier ou en ligne) remplis par les 200 cadres aux niveaux de surcharges hétérogènes}

Questionnaire 1 ère partie

$1^{\text {irs }}$ PARTIE : Renseignements utilisateur

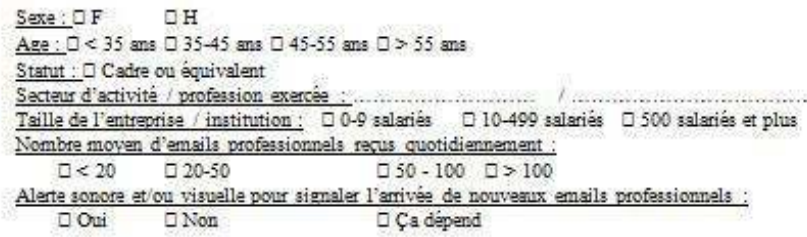




\section{Questionnaire $2^{\mathrm{e}}$ partie}

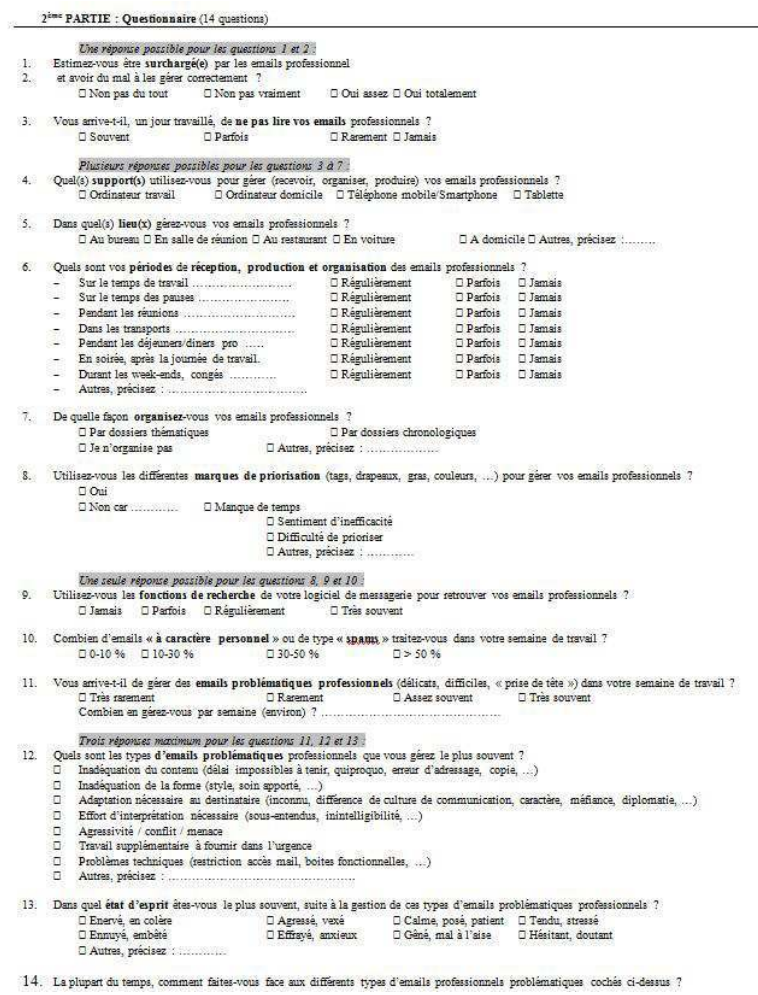

\section{NOTES}

1. Technologies de l'Information et de la Communication

2. Le stress est ici entendu comme un « état et un processus de l'ordre d'une transaction entre les ressources de l'individu et les contraintes situationnelles, estimées débordantes et menaçantes pour son bien-être, s'accompagnant de symptômes ou de dysfonctionnements physiques, psychologiques ou sociaux » (Lazarus \& Folkman, 1984).

3. Par analyse "située » des usages, nous entendons ici une approche qui puisse rendre compte de ce qui se passe réellement en contexte, de l'ancrage matériel et social de l'action, et de son caractère potentiellement opportuniste et improvisé en fonction du contexte (Salembier, Theureau, \& Relieu, 2004, pour leur analyse des travaux de Suchman).

4. Par analyse "écologique» des usages, nous entendons le recueil de données d'activités finalisées réalisées en conditions naturelles par des opérateurs volontaires. En psychologie ergonomique, nous opposons l'analyse écologique à l'analyse de laboratoire qui consiste à placer les individus dans un environnement et des conditions de réalisation d'activités simulées, expérimentales.

5. Pour plus de détails sur les intérêts et limites de la triangulation des méthodes dans cette étude, voir Créno et Cahour (2015).

6. Le questionnaire figure en Annexe 2.

7. Un exemple de grille d'observable est placé en Annexe 1.

8. Un exemple de journal de bord est présenté en Annexe 1.

9. Nous nous sommes inspirés de la technique d'entretien d'explicitation de Vermersch (1994) pour permettre aux cadres interviewés de décrire finement certaines situations d'activité bien spécifiées. Dans certains exemples, les verbatims renvoient à des explicitations de vécus très 
situés. Dans d'autres exemples, les verbatims sont plutôt d'ordre général, du fait de notre difficulté à amener quelques-uns de ces cadres en évocation. D'où notre appellation : entretiens « inspirés de l'entretien d'explicitation ».

10. La trame du questionnaire est présentée en Annexe 2.

11. Lazarus et Folkman (1984) définissent le coping comme l'ensemble des efforts cognitifs et comportementaux déployés pour gérer des exigences spécifiques internes et/ou externes qui sont évaluées par la personne comme consommant ou excédant ses ressources.

12. Lazarus et Folkman (1984) identifient trois types de stratégies : le coping centré sur le problème, qui vise à réduire les exigences de la situation ou à augmenter ses propres ressources pour mieux y faire face ; le coping centré sur l'émotion, qui comprend les tentatives pour réguler les tensions émotionnelles induites par la situation; et le coping centré sur le soutien social, qui consiste à obtenir la sympathie et l'aide d'autrui.

13. Fear Of Missing Out (FOMO) ou la peur de manquer une information, un évènement a été montrée par Przybylski, Murayam, DeHaan, \& Gradwell (2013) chez les jeunes générations qui se maintiennent connectées aux différents réseaux sociaux, sans jamais vivre pleinement la situation vécue en présentielle.

14. Voir l'étude de Mark et al. (2012) qui avait privé un panel d'usagers de Smartphone pendant 1 semaine afin d'évaluer les variations du taux de cortisol associés aux périodes de déconnexion.

15. Les cadres étudiés utilisaient l'un des deux logiciels de messagerie principaux du marché actuel : Outlook Express et Lotus Notes.

16. Colère lors de la lecture de certains emails, peur lors de la rédaction d'email sans règle ni repère.

\section{RÉSUMÉS}

L'article vise à comprendre les caractéristiques de l'activité de gestion des emails et les expériences vécues associées de cadres s'estimant surchargés par leurs emails. Une triangulation des méthodes a été mise en place pour parvenir à éclairer différents points de vue de l'activité. Des journées d'observation, des journaux de bord, des entretiens «resituants» et un questionnaire ont été menés auprès de deux panels de cadres surchargés volontaires. Cet article s'appuie sur la description détaillée de 4 cas de cadres particulièrement débordés par leurs emails, aux procédures de gestion et de régulation des emails différents. Les résultats décrivent les contextes socio-professionnels de ces cadres, le phénomène de gestion continue des emails leur permettant de tenter de faire face à l'explosion d'emails mais aussi les effets délétères sur leur santé et efficacité au travail. L'article souligne les spécificités des phases de lecture/ classement/rédaction d'emails, coûteuses cognitivement et émotionnellement pour ces cadres surchargés. L'identification d'emails «problématiques » et les émotions négatives associées sont apparues comme l'une des originalités de l'étude, marquant à nouveau le manque de repères et de règles communes aux usages hétérogènes du média. Enfin, des recommandations permettent de discuter des perspectives à donner à la messagerie électronique.

The article aims to understand the characteristics of email management activity and how managers swamped with emails cope with their experiences of this activity. Different methods were employed to gather different perspectives of the situated activity. Observation, diaries, indepth interviews and questionnaires were used on two panels of volunteer managers. This article 
is based on the description of 4 particularly overloaded managers with varying procedures for dealing with emails. The results describe their socio-professional contexts, their continuous daily connection and the harmful effects on their health and work efficiency. The article emphasizes the specificities of reading/filing/writing emails - processes that are cognitively and emotionally costly for these managers. It identifies 5 categories of "problematic" email and the negative emotions associated with them, underlining the lack of common rules between interlocutors. A certain number of recommendations are discussed to imagine the future of electronic messaging.

\section{INDEX}

Keywords : managers, email overload, activity, user experience, emotions, recommendations Mots-clés : cadres, surcharge d'emails, activité, expérience vécue, émotions, recommandations

\section{AUTEURS}

\section{LISA CRÉNO}

Département SES, Télécom ParisTech, 46, rue Barrault, 75013 Paris Cedex 13 lisa.creno@telecom-paristech.fr

\section{BÉATRICE CAHOUR}

Département SES, Télécom ParisTech, 46, rue Barrault, 75013 Paris cedex 13

beatrice.cahour@telecom-paristech.fr 